ECCOMAS

Proceedia
COMPDYN 2021

$8^{\text {th }}$ ECCOMAS Thematic Conference on Computational Methods in Structural Dynamics and Earthquake Engineering M. Papadrakakis, M. Fragiadakis (eds.) Streamed from Athens, Greece, 28 - 30 June 2021

\title{
DISPERSION ANALYSIS OF BULK ACOUSTIC WAVES IN PIEZOELECTRIC MEDIA DISCRETIZED BY ENERGY- ORTHOGONAL FINITE ELEMENTS
}

\author{
Francisco J. Brito Castro \\ Departamento de Ingeniería Industrial, Universidad de La Laguna \\ Calle Méndez Núñez 67-2C, Santa Cruz de Tenerife 38001, Spain \\ e-mail: fjbrito@ull.edu.es
}

\begin{abstract}
This contribution studies the propagation of bulk acoustic waves in piezoelectric media discretized by the finite element method. The element elastic and dielectric stiffness matrices are split into basic and higher order components which are related to the mean and deviatoric components of the element strain and electric fields, respectively. This decomposition is applied to the total energy of the finite element assemblage. By a dispersion analysis the higher order total energy is related to the total energy error for the propagating waves. An averaged correlation is proposed as an error indicator for the finite element vibration eigenmodes in piezoelectric solids.
\end{abstract}

Keywords: energy-orthogonal stiffness, numerical dispersion, piezoelectric media, vibration eigenmodes. 


\section{INTRODUCTION}

It is well known that the wave scattering at boundaries creates an interference field that, if composed solely of waves of frequency equal to a natural frequency of the bounded medium, takes the form of a standing-wave field which is an eigenmode of the continuum [1]. For homogeneous piezoelectric solids, whose three dimensions are comparable, this standing-wave field could be considered essentially composed of propagating quasi-longitudinal and quasishear bulk acoustic waves. In this case the goal of determining the finite element mesh required to accurately represent a given number of eigenmodes could be approached by analysing the effect of the spatial discretization over the propagation of such bulk acoustic waves in unbounded media. This effect becomes apparent by observing the dispersive behavior of the waves, a phenomenon that is not present in the physical system [2]. This contribution extends the author's previous research about homogeneous isotropic elastic solids [3].

The piezoelectric media covered in this research include PZT (Lead zirconate titanate $\left.\left(\mathrm{Pb}(\mathrm{Zr}, \mathrm{Ti}) \mathrm{O}_{3}\right)\right)$ piezoceramics [4]. These are transversely isotropic and they behave the same as crystals in class Hexagonal $(6 \mathrm{~mm})$. The poling direction is supposed along the Z-axis. The material constants for the piezoceramics selected (PZT-5H, PZT-8 and PZT-5A) can be found in reference [5]. Also PMN-PT (Lead magnesium niobate-lead titanate $\left(\mathrm{Pb}\left(\mathrm{Mg}_{1 / 3} \mathrm{Nb}_{2 / 3}\right) \mathrm{O}_{3}-\right.$ $\left.\mathrm{PbTiO}_{3}\right)$ ) <001> poled single crystals are covered [6]: the single crystal PMN-30PT from reference [7], and the commercial grade single crystals PMN-31PT and PIN-PMN-PT (TRS X2B and TRS X4B) from TRS Technologies Inc. [8] have been selected. These single crystals exhibit Tetragonal $(4 \mathrm{~mm})$ symmetry in macroscopic material properties. See Appendix 1 for the material constants of the PZT piezoceramics and PMN-PT single crystals selected.

An enlightening discussion about PZT piezoceramics and PMN-PT single crystals in the context of sonar applications can be found in reference [9].

\subsection{Finite element formulation}

In a lossless piezoelectric medium discretized by the finite element method the equations of equilibrium governing its linear dynamic response for time-harmonic waves will be [10],

$$
\left(\begin{array}{cc}
\mathbf{K}_{u u}-\omega^{2} \mathbf{M} & \mathbf{K}_{u \phi} \\
-\mathbf{K}_{\phi u} & \mathbf{K}_{\phi \phi}
\end{array}\right)\left(\begin{array}{l}
\mathbf{x} \\
\boldsymbol{\phi}
\end{array}\right)=\left(\begin{array}{l}
\mathbf{F} \\
\mathbf{Q}
\end{array}\right), \quad \mathbf{K}_{\phi u}=\mathbf{K}_{u \phi}^{T} ; \begin{array}{ll}
\mathbf{x}=\tilde{\mathbf{x}} \exp (-i \omega t), & \mathbf{F}=\tilde{\mathbf{F}} \exp (-i \omega t) \\
\boldsymbol{\phi}=\tilde{\boldsymbol{\phi}} \exp (-i \omega t), & \mathbf{Q}=\tilde{\mathbf{Q}} \exp (-i \omega t)
\end{array}
$$

where: $\mathbf{M}$, consistent mass matrix; $\mathbf{K}_{u u}\left(\mathbf{K}_{\phi \phi}, \mathbf{K}_{u \phi}\right)$, elastic (dielectric, piezoelectric) stiffness matrix of the finite element assemblage; $\tilde{\mathbf{x}}(\tilde{\mathbf{F}}, \tilde{\boldsymbol{\phi}}, \tilde{\mathbf{Q}})$, column matrix containing the complex amplitude of the nodal displacements (external loads, electric potentials, free electric charges); $\omega=2 \pi / T$, circular frequency; $T$, period of wave; $t$, time.

The total energy will be equal to the sum of pure elastic energy and pure electric energy [11],

$$
E^{M}=\frac{1}{2} \operatorname{Re}\left[\mathbf{x}^{T}\right] \mathbf{K}_{u u} \operatorname{Re}[\mathbf{x}] \quad(a) \quad E^{E}=\frac{1}{2} \operatorname{Re}\left[\boldsymbol{\phi}^{T}\right] \mathbf{K}_{\phi \phi} \operatorname{Re}[\boldsymbol{\phi}] \quad(b)
$$

At element level

$$
\begin{array}{rll}
\mathbf{K}_{u u}^{e}=\int_{V e} \mathbf{B}_{u}^{T}\left[\mathbf{C}^{E}\right] \mathbf{B}_{u} d V & (a) & \mathbf{K}_{\phi \phi}^{e}=\int_{V e} \mathbf{B}_{\phi}^{T}\left[\boldsymbol{\varepsilon}^{S}\right] \mathbf{B}_{\phi} d V \\
\mathbf{K}_{u \phi}^{e}=\int_{V e} \mathbf{B}_{u}^{T}[\mathbf{e}]^{T} \mathbf{B}_{\phi} d V & (c) & \mathbf{K}_{\phi u}^{e}=\int_{V e} \mathbf{B}_{\phi}^{T}[\mathbf{e}] \mathbf{B}_{u} d V
\end{array}
$$

where: $\mathbf{B}_{u}\left(\mathbf{B}_{\phi}\right)$, matrix relating the strain components (gradient of electric potential) to the nodal values of displacement (electric potential); $\mathbf{C}^{\mathrm{E}}$, elasticity matrix evaluated at constant electric field; $\boldsymbol{\varepsilon}^{\mathrm{S}}$, dielectric matrix evaluated at constant mechanical strain; e, piezoelectric matrix. 
If the matrices $\mathbf{B}_{u}$ and $\mathbf{B}_{\phi}$ are partitioned into mean and deviatoric components,

$$
\begin{array}{llll}
\mathbf{B}_{u}=\overline{\mathbf{B}}_{u}+\mathbf{B}_{u, d} ; & \overline{\mathbf{B}}_{u} V^{e}=\int_{V e} \mathbf{B}_{u} d V, & \mathbf{B}_{u, d}=\mathbf{B}-\overline{\mathbf{B}}_{u} & (a) \\
\mathbf{B}_{\phi}=\overline{\mathbf{B}}_{\phi}+\mathbf{B}_{\phi, d} ; & \overline{\mathbf{B}}_{\phi} V^{e}=\int_{V e} \mathbf{B}_{\phi} d V, & \mathbf{B}_{\phi, d}=\mathbf{B}-\overline{\mathbf{B}}_{\phi} \quad(b)
\end{array}
$$

the matrices Eq. (3a) and Eq. (3b) would be respectively decomposed as addition of basic and higher order components,

$$
\begin{array}{rll}
\mathbf{K}_{u u}^{e} & =\mathbf{K}_{u u, b}^{e}+\mathbf{K}_{u u, h}^{e} ; \quad \mathbf{K}_{u u, b}^{e}=\overline{\mathbf{B}}_{u}^{T}\left[\mathbf{C}^{E}\right] \overline{\mathbf{B}}_{u} V^{e}, & \mathbf{K}_{u u, h}^{e}=\int_{V e} \mathbf{B}_{u, d}^{T}\left[\mathbf{C}^{E}\right] \mathbf{B}_{u, d} d V \\
\mathbf{K}_{\phi \phi}^{e} & =\mathbf{K}_{\phi \phi, b}^{e}+\mathbf{K}_{\phi \phi, h}^{e} ; \quad \mathbf{K}_{\phi \phi, b}^{e}=\overline{\mathbf{B}}_{\phi}^{T}\left[\boldsymbol{\varepsilon}^{S}\right] \overline{\mathbf{B}}_{\phi} V^{e}, & \mathbf{K}_{\phi \phi, h}^{e}=\int_{V e} \mathbf{B}_{\phi, d}^{T}\left[\boldsymbol{\varepsilon}^{S}\right] \mathbf{B}_{\phi, d} d V
\end{array}
$$

In this case it is said that the element stiffness matrices are formulated in energy-orthogonal form [12]. The decomposition in Eq. (5) holds for the complete model,

$$
\mathbf{K}_{u u}=\mathbf{K}_{u u, b}+\mathbf{K}_{u u, h} \quad(a) \quad \mathbf{K}_{\phi \phi}=\mathbf{K}_{\phi \phi, b}+\mathbf{K}_{\phi \phi, h}
$$

For a stationary wave the amplitudes of nodal displacements and electric potentials are realvalued vectors in Eq. (1). Then, from Eq. (2a) and Eq. (2b), the period-averaged elastic and electric energies for the discretized domain will be, respectively,

$$
\bar{E}^{M}=\frac{1}{4} \tilde{\mathbf{x}}^{T} \mathbf{K}_{u u} \tilde{\mathbf{x}} \quad(a) \quad \bar{E}^{E}=\frac{1}{4} \tilde{\boldsymbol{\phi}}^{T} \mathbf{K}_{\phi \phi} \tilde{\boldsymbol{\phi}}
$$

By introducing Eq. (6) into Eq. (7), the basic and higher order period-averaged energies will be obtained. The latter components will be

$$
\bar{E}_{h}^{M}=\frac{1}{4} \tilde{\mathbf{x}}^{T} \mathbf{K}_{u u, h} \tilde{\mathbf{x}} \quad(a) \quad \bar{E}_{h}^{E}=\frac{1}{4} \tilde{\boldsymbol{\phi}}^{T} \mathbf{K}_{\phi \phi, h} \tilde{\boldsymbol{\phi}} \quad(b)
$$

\section{DISPERSION ANALYSIS}

The unbounded piezoelectric domain is discretized by a regular mesh of finite elements. Two different isoparametric finite elements with consistent mass matrix are considered: the hexahedron with twenty nodes and brick geometry HE20, Fig. 1, and the pentahedron with fifteen nodes and right prismatic isosceles geometry PE15, Fig. 2 [13]. The nodal lattice formed by the finite element assemblage has four and five nodes per unit cell, respectively. Different meshes with the same element volume can be selected by the aspect ratio parameter, $0<\gamma \leq 1$; the skew angle, $0 \leq \beta<90^{\circ}$ (only HE 20 element); and the distortion-Z parameter, $\varepsilon>0$.

For uniform plane harmonic bulk waves at the continuum piezoelectric medium, the displacement vector field and the electric potential field will be, respectively,

$$
\mathbf{u}=A^{u} \hat{\mathbf{a}} \exp (i \kappa \mathbf{n} \cdot \mathbf{r}) \exp (-i \omega t) \quad(a) \quad \phi=A^{\phi} \exp (i \kappa \mathbf{n} \cdot \mathbf{r}) \exp (-i \omega t)
$$

where: $A^{u}$ and $A^{\phi}$, amplitudes of the wave; â, polarization vector; $\mathbf{n}$, wave normal, unit vector indicating the direction of the wave propagation; $\mathbf{r}$, position vector; $\kappa=2 \pi / \lambda=\omega / c$, wave number; $\lambda$, wavelength; $c$, phase velocity at the continuum medium.

The wave normal has the components $n_{1}=\cos \phi \sin \theta, n_{2}=\sin \phi \sin \theta$ and $n_{3}=\cos \theta$, where: $\phi$, azimuthal angle, $0 \leq \phi \leq 180^{\circ} ; \theta$, polar angle, $0 \leq \theta \leq 180^{\circ}$, Fig. 1 .

The polarization vectors and phase velocities are solution of the stiffened Christoffel equation, which is expressed in abbreviated subscript notation [14],

$$
\left(l_{i K}\left(C_{K L}^{E}+\left[e_{K j} n_{j}\right]\left[n_{i} e_{i L}\right] /\left(n_{i} \varepsilon_{i j}^{S} n_{j}\right)\right) l_{L j}\right) \hat{a}_{j}=\rho c^{2} \hat{a}_{i}, \quad i, j=1, \ldots, 3 ; \quad K, L=1, \ldots, 6
$$


where the non-null values of $l_{i K}$ are: $l_{11}=n_{1}, l_{15}=n_{3}, l_{16}=n_{2}, l_{22}=n_{2}, l_{24}=n_{3}, l_{26}=n_{1}, l_{33}=n_{3}$, $l_{34}=n_{2}$, and $l_{35}=n_{1}$.
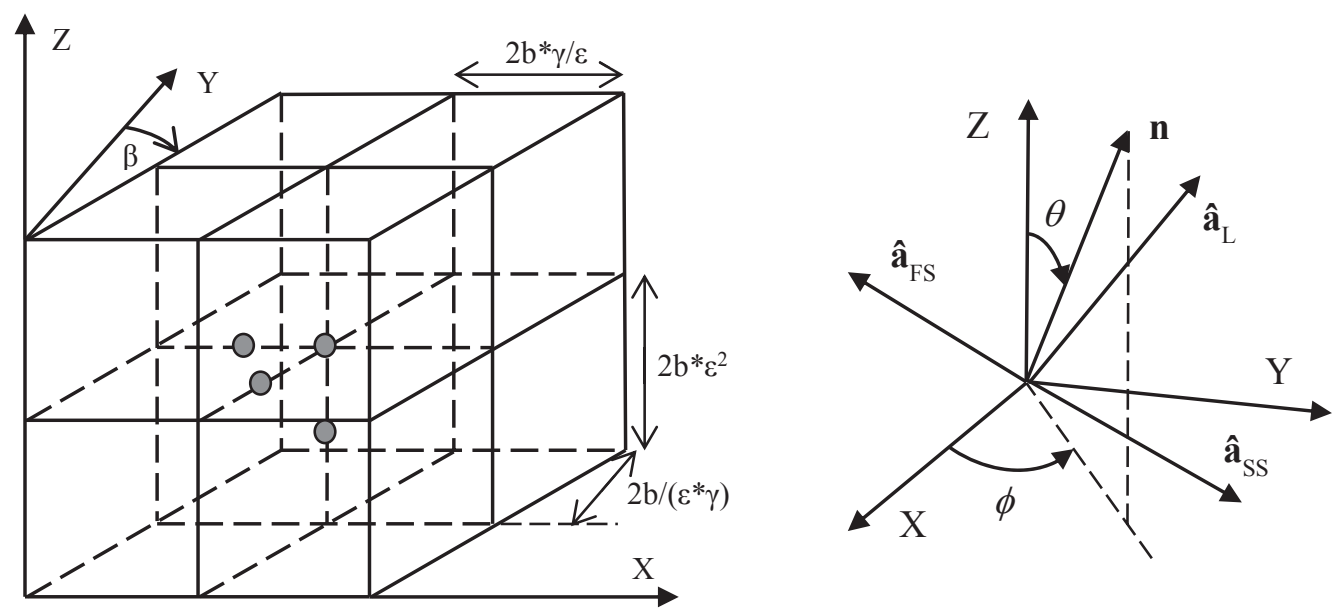

Figure 1: Piezoelectric domain discretized by a regular mesh of HE20 elements and unit cell with four nodes. Polarization vectors.

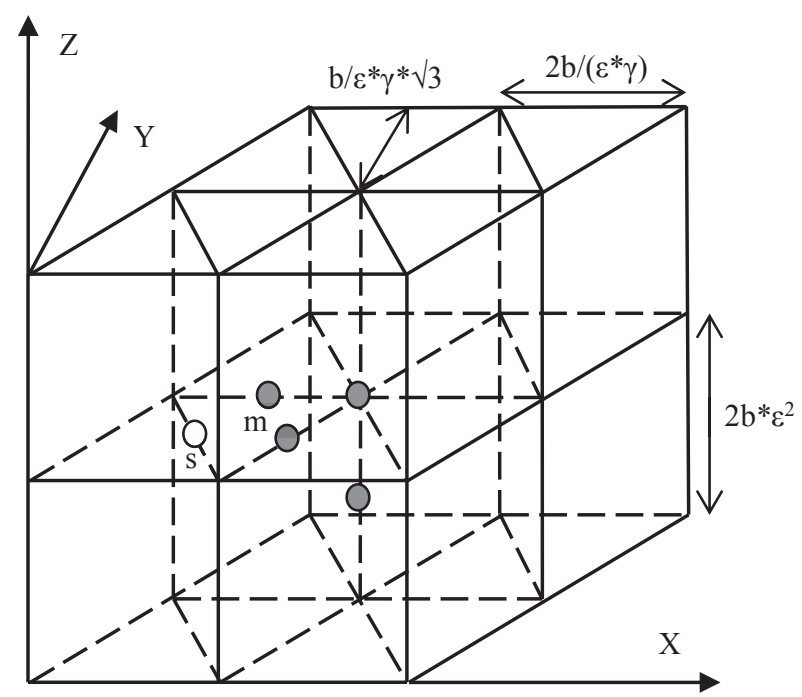

Figure 2: Piezoelectric domain discretized by a regular mesh of PE15 elements and unit cell with four master nodes $(\mathrm{m})$ and one slave node $(\mathrm{s})$.

By solving the eigenproblem Eq. (10), three mutually orthogonal waves at the continuum medium are computed, Fig. 1: ( $\left.\hat{\mathbf{a}}_{S S}, \mathrm{c}_{S S}\right)$, slow quasi-shear wave, SS; $\left(\hat{\mathbf{a}}_{F S}, \mathbf{c}_{F S}\right)$, fast quasi-shear wave, FS; and $\left(\hat{\mathbf{a}}_{L}, \mathrm{c}_{L}\right)$, quasi-longitudinal wave, $\mathrm{L}$.

Then, the electric potential at the continuum medium can be computed for each wave,

$$
A^{\phi}=A^{u}\left(n_{i} e_{i K} l_{K j} /\left(n_{i} \varepsilon_{i j}^{S} n_{j}\right) \hat{a}_{j}\right), \quad i, j=1, \ldots, 3 ; \quad K=1, \ldots, 6
$$

For the displacement vector field Eq. (9a) and for the electric potential field Eq. (9b), the densities of period-averaged pure elastic energy and period-averaged pure electric energy at the continuum medium can be respectively computed by the equations [14]

$$
\bar{E}_{0}^{M}=\frac{1}{4} \omega^{2}\left(A^{u}\right)^{2} l_{K i} \hat{a}_{i} C_{K L}^{E} l_{L j} \hat{a}_{j} / c^{2} \quad \text { (a) } \bar{E}_{0}^{E}=\frac{1}{4} \omega^{2}\left(A^{\phi}\right)^{2} n_{i} \varepsilon_{i j}^{S} n_{j} / c^{2}
$$




\subsection{Characteristic equations}

The characteristic equations can be found assuming harmonic waves Eq. (9) with different amplitudes in each node of the unit cell,

$$
\tilde{\mathbf{u}}=A_{j}^{u} \hat{\mathbf{a}} \exp (i \kappa \mathbf{n} \cdot \mathbf{r}) \quad(a) \quad \tilde{\phi}=A_{j}^{\phi} \exp (i \kappa \mathbf{n} \cdot \mathbf{r}) \quad(b) ; \quad j=1, \ldots, N
$$

where: $N$, number of nodes per unit cell.

Inserting the solutions Eq. (13) into the homogeneous part of Eq. (1), the characteristic equation for each node of the unit cell is yielded by equilibrium of nodal forces into the direction of the particle displacement [15],

$$
\mathbf{F}_{K}^{u u} \cdot \hat{\mathbf{a}}-\omega^{2} \mathbf{F}_{M} \cdot \hat{\mathbf{a}}+\mathbf{F}_{K}^{u \phi} \cdot \hat{\mathbf{a}}=0, \quad-F_{K}^{\phi u}+F_{K}^{\phi \phi}=0
$$

By considering Eq. (14) for each node of the unit cell, a homogeneous system of $2 \mathrm{~N}$ algebraic equations is formed,

$$
\begin{gathered}
\left(\begin{array}{cc}
\mathbf{a}^{u u}(m, \phi, \theta, \boldsymbol{\alpha})+\varpi^{2} \mathbf{b}^{u u}(m, \phi, \theta, \boldsymbol{\alpha}) & \mathbf{a}^{u \phi}(m, \phi, \theta, \boldsymbol{\alpha}) \\
\mathbf{a}^{\phi u}(m, \phi, \theta, \boldsymbol{\alpha}) & \mathbf{a}^{\phi \phi}(m, \phi, \theta, \boldsymbol{\alpha})
\end{array}\right)\left(\begin{array}{l}
\mathbf{A}^{u} \\
\mathbf{A}^{\phi}
\end{array}\right)=\left(\begin{array}{l}
\mathbf{0} \\
\mathbf{0}
\end{array}\right) \\
m=b \kappa / \pi=2 b / \lambda, \quad \varpi=(2 b / c) \omega
\end{gathered}
$$

where: $m$, dimensionless wave number, $0<m<1$; $b$, half of the element size; $\varpi$, dimensionless frequency of the discretized piezoelectric medium; $\boldsymbol{\alpha}=(\gamma, \beta, \varepsilon)$ form parameter for HE20 mesh, or $\boldsymbol{\alpha}=(\gamma, \varepsilon)$ form parameter for PE15 mesh.

In this procedure both the global stiffness matrices and the global mass matrix have been expressed in a suitable form,

$$
\mathbf{K}_{x x}=\rho c^{2}(2 b) \mathbf{K}_{x x}^{0} \quad x x=(u u, u \phi, \phi u, \phi \phi), \quad \mathbf{M}=\rho(2 b)^{3} \mathbf{M}^{0}
$$

The homogeneous system of $2 N$ algebraic equations Eq. (15) can be reduced to a homogeneous system of $N$ equations by static condensation [16]. So, from Eq. (15),

$$
\left(\mathbf{a}^{u u}+\varpi^{2} \mathbf{b}^{u u}\right) \mathbf{A}^{u}+\mathbf{a}^{u \phi} \mathbf{A}^{\phi}=\mathbf{0} \quad(a) \quad \mathbf{A}^{\phi}=-\left(\mathbf{a}^{\phi \phi}\right)^{-1} \mathbf{a}^{\phi u} \mathbf{A}^{u} \quad(b)
$$

Inserting Eq. (18b) into (18a), the condensate system of characteristic equations is formed,

$$
\left(\mathbf{a}^{u u}-\mathbf{a}^{u \phi}\left(\mathbf{a}^{\phi \phi}\right)^{-1} \mathbf{a}^{\phi u}+\varpi^{2} \mathbf{b}^{u u}\right) \mathbf{A}^{u}=\mathbf{0} \quad \Leftrightarrow \quad \mathbf{Z A}^{u}=\mathbf{0}
$$

\subsection{Dispersion equations}

The system of homogeneous algebraic equations given in Eq. (19) has a non-trivial solution only if the matrix $\mathbf{Z}$ is singular; that is, $\operatorname{det}[\mathbf{Z}]=0$. Then it is yielded the following polynomial equation which is called the characteristic frequency equation for the plane wave propagation,

$$
\sum_{r=0}^{N} c_{r}(m, \phi, \theta, \boldsymbol{\alpha}) \varpi^{2 r}=0, \quad c_{N}=1
$$

It is an important fact that the $N$ zeroes of a polynomial of degree $N \geq 1$ with complex coefficients depend continuously upon the coefficients [17]. Thus, sufficiently small changes in the coefficients of a polynomial can lead only to small changes in any zero. However, if the zeros are numerically computed, there is no simple way to define a function which takes the $N$ coefficients (all but the leading 1) of a monic polynomial of degree $N$ to the $N$ zeroes of the polynomial, since there is no natural way to define an ordering among the $N$ zeroes. In the case of the HE20 mesh, for which the polynomial Eq. (20) is quartic, the above difficulty has been 
overcome by computing the zeroes in closed form as functions of its coefficients. Then, the components

$$
\varpi_{k}=\varpi_{k}(m, \phi, \theta, \boldsymbol{\alpha}), \quad k=1, \ldots, 4
$$

will be continuous functions precisely defined. They are called the dispersion equations.

Substituting Eq. (21) into Eq. (19), the displacement wave amplitudes corresponding to the nodes of the unit cell are yielded for each dispersion equation. Then, by Eq. (18b), the corresponding electric potential amplitudes are recovered.

To find the zeros of a polynomial equation as functions of its coefficients beyond the quartic equation is a very difficult mathematical problem [18]. Then, for the mesh PE15 which has five nodes per unit cell, if the zeros of Eq. (20) are numerically computed, the above mentioned ordering difficulty could be a problem. In this case, by considering the initial condition,

$$
\lim _{m \rightarrow 0} \varpi_{1}(m, \phi, \theta, \boldsymbol{\alpha})=0
$$

it is proposed to compute the first dispersion equation by a reduced unit cell obtained by a procedure of exact dynamic condensation [16].

Assume that the total nodes at the unit cell are categorized as master nodes $(\mathrm{m})$ and slave nodes (s), where the number of master nodes is four and the number of slave nodes is one, Fig 2. With this arrangement, the system of characteristic equations Eq. (19) may be partitioned as

$$
\left(\begin{array}{cc}
\mathbf{Z}_{m m}(q) & \mathbf{Z}_{m s}(q) \\
\mathbf{Z}_{s m}(q) & \mathbf{Z}_{s s}(q)
\end{array}\right)\left(\begin{array}{c}
\mathbf{A}_{m}^{u} \\
\mathbf{A}_{s}^{u}
\end{array}\right)=\left(\begin{array}{l}
\mathbf{0} \\
\mathbf{0}
\end{array}\right), \quad q=\varpi^{2}
$$

From Eq. (23) the relation of wave amplitudes between the master and slave nodes may be obtained as

$$
\mathbf{A}_{s}^{u}=-\mathbf{Z}_{s s}^{-1}(q) \mathbf{Z}_{s m}(q) \mathbf{A}_{m}^{u}
$$

Then, by back-substituting, the system of characteristic equations of the reduced unit cell is obtained as

$$
\mathbf{Z}_{R}(q) \mathbf{A}_{m}^{u}=\mathbf{0}, \quad\left[\mathbf{K}_{R}(q)+q \mathbf{M}_{R}(q)\right] \mathbf{A}_{m}^{u}=\mathbf{0}
$$

Then, from Eq. (25), the reduced form of the characteristic frequency equation is obtained

$$
q^{4}+c_{3 R} q^{3}+c_{2 R} q^{2}+c_{1 R} q+c_{0 R}=0, \quad c_{r R}(m, \phi, \theta, \boldsymbol{\alpha}, q), \quad r=0, \ldots, 3
$$

The first dispersion equation is computed by the following iterative procedure: Set by Eq. (22) the initial value $q_{i 1}=0$.

Do for $0<m<1$, step $\Delta m$

1. Compute the coefficients $c_{r R}\left(m, \phi, \theta, \boldsymbol{\alpha}, q_{i 1}\right)$ of Eq. (26).

2. Compute the zero $q_{1}$ of the quartic polynomial Eq. (26).

Do while $\left(\left|\left(q_{1}-q_{i 1}\right) / q_{1}\right| \geq \delta\right)$

1. Refresh initial value $q_{i 1}=q_{1}$.

2. Compute the coefficients $c_{r R}\left(m, \phi, \theta, \boldsymbol{\alpha}, q_{i 1}\right)$ of Eq. (26).

3. Compute the zero $q_{1}$ of the quartic polynomial Eq. (26).

\section{End do}

3. Substituting $q_{1}$ into Eq. (25) to compute the wave amplitudes at the master nodes. 
4. Substituting $q_{1}$ into Eq. (24) to compute the wave amplitudes at the slave nodes.

5. Refresh initial value $q_{i 1}=q_{1}$ for the next step.

\section{End do}

It must be recall the equality of the energy and group velocities for bulk acoustic waves in a lossless piezoelectric medium [19], and the equality of the normal component of the group velocity and the phase velocity at the anisotropic continuum [20]. Then, the indicators of numerical dispersion for the phase velocity and the normal component of the group velocity are yielded for each dispersion equation. From Eq. (16),

$$
e_{p}=c_{p} / c=(2 \pi)^{-1} \varpi / m \quad(a) \quad e_{g n}=c_{g n} / c=(2 \pi)^{-1} \partial \varpi / \partial m \quad(b)
$$

The range of dimensionless wave number values where each dispersion equation represents the propagation of acoustic waves in the discretized medium will be called the acoustical branch of the dispersion equation. In order to determine the acoustical branches the following constraint conditions are imposed,

$$
A_{1}^{u}=1, \quad A_{j}^{u}(m, \phi, \theta, \boldsymbol{\alpha})>0 \quad j=2, \ldots, N \quad(a) \quad(\partial \varpi / \partial m)_{\phi, \theta, \boldsymbol{\alpha}}>0
$$

In molecular physics the condition Eq. (28a) is called the restriction of the lattice spectrum to the acoustical branch [21]. By considering Eq. (27b), the constraint condition Eq. (28b) imposes that the normal component of the group velocity or energy velocity must have the wave direction. The preliminary constraint condition $\operatorname{dim}[\mathbf{N}(\mathbf{Z})]=1$, over the dimension of the null space of matrix $\mathbf{Z}$ for the HE20 mesh, or $\operatorname{dim}\left[\mathbf{N}\left(\mathbf{Z}_{\mathrm{R}}\right)\right]=1$ for the PE15 mesh, must be imposed in order to Eq. (28a) would be a meaningful constraint condition. For each dispersion equation only the acoustical branch will be considered.

\subsection{Energy at the unit cell}

From Eq. (2), Eq. (16) and Eq. (17), the densities of period-averaged elastic and electric energies at the unit cell are computed,

$$
\begin{aligned}
& \bar{E}^{M}=\frac{1}{2} \rho(\omega / \varpi)^{2} C \int_{0}^{1} \operatorname{Re}\left[\tilde{\mathbf{x}}^{T} \exp (-i 2 \pi \tau)\right] \operatorname{Re}\left[\tilde{\mathbf{F}}_{u u}^{0} \exp (-i 2 \pi \tau)\right] d \tau \quad \text { (a) } \\
& \bar{E}^{E}=\frac{1}{2} \rho(\omega / \varpi)^{2} C \int_{0}^{1} \operatorname{Re}\left[\tilde{\boldsymbol{\phi}}^{T} \exp (-i 2 \pi \tau)\right] \operatorname{Re}\left[\tilde{\mathbf{F}}_{\phi \phi}^{0} \exp (-i 2 \pi \tau)\right] d \tau \quad \text { (b) }
\end{aligned}
$$

where: $\tau=t / T, 0 \leq \tau \leq 1$, dimensionless time; $\tilde{\mathbf{F}}_{u u}^{0}$ and $\tilde{\mathbf{F}}_{\phi \phi}^{0}$ are column matrices of forces at the nodes of the unit cell; $\mathrm{C}=1$ for the HE20 mesh, $\mathrm{C}=2 /(3)^{1 / 2}$ for the PE15 mesh.

From the decomposition in Eq. (6), the above computed densities of period-averaged energies Eq. (29) can be partitioned as addition of basic and higher order components. Then, the percentage of period-averaged higher order total energy can be defined as

$$
e_{h}=\left(\bar{E}_{h}^{M}+\bar{E}_{h}^{E}\right) /\left(\bar{E}^{M}+\bar{E}^{E}\right)=\bar{E}_{h} / \bar{E}, \quad e_{h}=e_{h}(m, \phi, \theta, \boldsymbol{\alpha})
$$

From Eq. (29) and Eq. (12), the percentage indicator of total energy error associated with the spatial discretization that is introduced by the finite element model is defined as

$$
e_{T}=\left(\left(\bar{E}^{M}+\bar{E}^{E}\right) /\left(\bar{E}_{0}^{M}+\bar{E}_{0}^{E}\right)\right)-1=\left(\bar{E} / \bar{E}_{0}\right)-1, \quad e_{T}=e_{T}(m, \phi, \theta, \boldsymbol{\alpha})
$$

From Eq. (30) and Eq. (31), a mapping between the total energy error and the percentage of higher order total energy can be also computed,

$$
e_{T}=e_{T}\left(e_{h}, \phi, \theta, \boldsymbol{\alpha}\right)
$$




\subsection{Substitute wave field}

It is clear that the harmonic acoustic waves cannot be exactly captured by a regular mesh of finite elements HE20 or PE15. This fact is a consequence of the element interpolation which is quadratic. A substitute wave field [22] or alias field [13] has been obtained in the discretized unbounded medium by performing a dispersion analysis in terms of allowable polarizations of the continuum. The substitute wave field is obtained by collocating Eq. (13a) and Eq. (13b) in each node of the unit cell. The assumption of different amplitudes is introduced because the equation of equilibrium is different for each node. The analysis yields the relative wave amplitudes and the dispersion relation under which the alias field may propagate in the discretized medium. The alias wave field will be

$$
\tilde{\mathbf{u}}^{a}=\sum N_{S, i} \tilde{\mathbf{u}}_{i}, \quad \tilde{\phi}^{a}=\sum N_{S, i} \tilde{\phi}_{i}
$$

where: $\widetilde{\mathbf{u}}_{i}$, the values of Eq. (13a) at the nodes; $\tilde{\phi}_{i}$, the values of Eq. (13b) at the nodes; $N_{S, i}$, the nodal shape functions.

By computing the wave amplitude distortion, the discretization error of the wave field Eq. (33) can be decomposed as addition of interpolation and pollution errors [23].

\subsection{Numerical research}

For the waves and media considered the indicators Eq. (27), Eq. (30) and Eq. (31) are computed versus dimensionless wave number for different meshes and directions of wave propagation. Five meshes having the same element volume will be considered for each of the elements analyzed. Specifically, for HE20 element, Fig. 1:

Q1: square section; $\gamma=1, \beta=0, \varepsilon=1$.

Q2: rectangular section with aspect ratio $1: 2 ; \gamma=1 / \sqrt{ } 2, \beta=0, \varepsilon=1$.

Q3: skewed section; $\gamma=1, \beta=45^{\circ}, \varepsilon=1$.

Q4: distorted in the poling Z-direction with aspect ratio 2:1; $\gamma=1, \beta=0, \varepsilon=2^{1 / 3}$.

Q5: distorted in the poling Z-direction with aspect ratio $1: 2 ; \gamma=1, \beta=0, \varepsilon=1 / 2^{1 / 3}$.

and, for PE15 element, Fig. 2:

T1: triangular section of equilateral geometry; $\gamma=1, \varepsilon=1$.

T2: triangular section of right geometry; $\gamma^{2}=1 /(3)^{1 / 2}, \varepsilon=1$.

T3: triangular section with angle of $30^{\circ}$ opposite to the base; $\gamma^{2}=1 /(3)^{1 / 2} \operatorname{tg} 75^{\circ}, \varepsilon=1$.

T4: distorted in the poling Z-direction with aspect ratio $2: 1 ; \gamma=1, \varepsilon=2^{1 / 3}$.

T5: distorted in the poling Z-direction with aspect ratio $1: 2 ; \gamma=1, \varepsilon=1 / 2^{1 / 3}$.

Given the mesh and the piezoelectric medium, the dispersion analysis is numerically carried out by a step of $\pi / 36$ for the azimuthal and polar angles, and a step of $1 / 10000$ for the dimensionless wave number. As result the indicators Eq. (27), Eq. (30) and Eq. (31), are computed as finite sets of values. An example of the indicators Eq. (30) and Eq. (32) is shown in Fig. 3. It is observed that Eq. (30) and it is deduced that Eq. (31) both vanish as dimensionless wave number goes to zero; that is, as the mesh is refined and in the limit of long waves. It must be remarked that the behavior of the higher order total energy as dimensionless wave number goes to zero is a consequence that both the strain field and the electric field inside each element becomes uniform. That is, given the mesh, in the limit of long waves, the density of total energy 
approaches to zero more slowly than its higher order component; and, given the wavelength, as the solution converges on account of mesh refinement, the density of total energy is increasingly dominated by its basic component.

Investigating the relationship between the total energy error and the percentage of higher order total energy Eq. (32), it can be observed from Fig. 3 that both variables could be related by a cubic function for moderate values of the higher order total energy,

$$
e_{T}=\left[A(\phi, \theta, \boldsymbol{\alpha}) * e_{h}+B(\phi, \theta, \boldsymbol{\alpha})\right] * e_{h}^{2}
$$

For each of the waves (SS, FS, and L) and piezoelectric media considered in this research, an averaged correlation between the total energy error and the percentage of higher order total energy is sought by computing averaged values for the coefficients $A$ and $B$.

First, two reference values of the percentage of higher order total energy are selected,

$$
\text { HE20: } e_{h 1}=0.10 \quad e_{h 2}=0.20 ; \text { PE15: } e_{h 1}=0.075 \quad e_{h 2}=0.15
$$
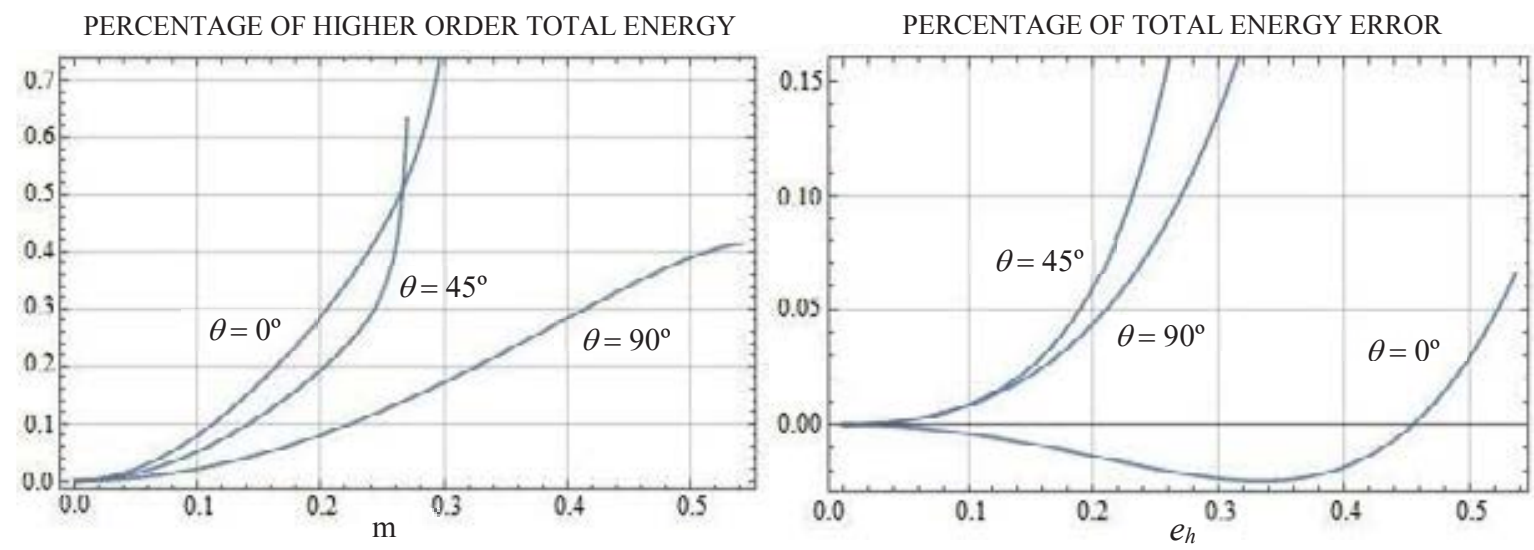

Figure 3: Percentage of higher order total energy versus dimensionless wave number and versus percentage of total energy error. PMN-30PT, MESH Q4, L-WAVES, $\phi=45^{\circ}$.

Then, by Eq. (30) and Eq. (32), the reference values related of dimensionless wave number and percentage of total energy error are respectively computed,

$$
m_{1,2}=m_{1,2}(\phi, \theta, \boldsymbol{\alpha}), \quad e_{T 1,2}=e_{T 1,2}(\phi, \theta, \boldsymbol{\alpha})
$$

The mean value of each reference dimensionless wave number, and the root mean square value (based on the $\mathrm{L}_{2}$-norm) and the sup-norm value [24] of each reference total energy error are next computed on the range of propagation angles,

$$
\begin{gathered}
m_{1,2}^{M}(\boldsymbol{\alpha})=\frac{1}{\pi^{2}} \int_{0}^{\pi} \int_{0}^{\pi} m_{1,2} d \phi d \theta \quad(a) \quad e_{T 1,2}^{R M S}(\boldsymbol{\alpha})=\sqrt{\frac{1}{\pi^{2}} \int_{0}^{\pi} \int_{0}^{\pi}\left|e_{1,2}\right|^{2} d \phi d \theta} \\
e_{T 1,2}^{S U P}(\boldsymbol{\alpha})=\sup \left\{\left|e_{1,2}(\phi, \theta, \boldsymbol{\alpha})\right|: \quad 0 \leq \phi, \theta \leq \pi\right\} \quad(c)
\end{gathered}
$$

Consistent with the discrete analysis carried out, the integrals in Eq. (37a) and Eq. (37b) are numerically computed. Mean values of the indicators of numerical dispersion for the phase velocity and the normal component of the group velocity Eq. (27) have been also computed. Detailed values are presented in the Appendix 2 attached to this manuscript.

As a next step, the values computed in Eq. (37a) and Eq. (37b) are mesh-averaged, 


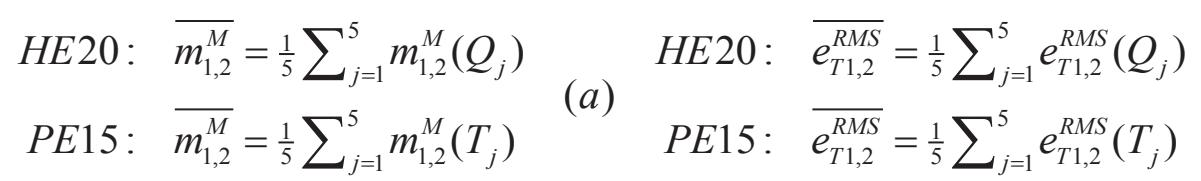

The results of Eq. (38a) and Eq. (38b) are displayed in Table 1 and Table 2, respectively, for each of the piezoelectric media and waves investigated. Similar mesh-averaged values of the indicators of numerical dispersion for the phase velocity and the normal component of the group velocity Eq. (27) have been also computed, Table 3.

From Table 1 and Table 2, it is deduced that the mesh-averaged root mean square values of the first and the second reference percentage of total energy error roughly correspond, in an averaged sense, to six and four elements per wavelength, respectively. In such averaged sense, the total energy error of the quasi-longitudinal waves is greater than the one of the quasi-shear waves. An opposite behavior is shown by the indicators of numerical dispersion. It is deduced from Table 3 that the numerical dispersion of the quasi-shear waves is greater than the one of the quasi-longitudinal waves.

\begin{tabular}{|c|c|c|c|c|c|c|c|}
\hline & & \multicolumn{3}{|c|}{ HE20 } & \multicolumn{3}{|c|}{ HE20 } \\
\hline & & PZT-5H & PZT-8 & PZT-5A & PMN-30PT & PMN-31PT & PIN-PMN-PT \\
\hline \multirow{3}{*}{$\overline{m_{1}^{M}}$} & $\mathrm{SS}$ & 0.1758 & 0.1756 & 0.1755 & 0.1751 & 0.1753 & 0.1753 \\
\hline & FS & 0.1756 & 0.1758 & 0.1759 & 0.1754 & 0.1754 & 0.1754 \\
\hline & $\mathrm{L}$ & 0.1748 & 0.1749 & 0.1748 & 0.1745 & 0.1745 & 0.1745 \\
\hline \multirow{5}{*}{$\overline{\overline{m_{2}^{M}}}$} & SS & 0.2576 & 0.2571 & 0.2567 & 0.2553 & 0.2558 & 0.2557 \\
\hline & FS & 0.2568 & 0.2574 & 0.2577 & 0.2564 & 0.2563 & 0.2563 \\
\hline & $\mathrm{L}$ & 0.2542 & 0.2545 & 0.2543 & 0.2530 & 0.2531 & 0.2532 \\
\hline & & \multicolumn{3}{|c|}{ PE15 } & \multicolumn{3}{|c|}{ PE15 } \\
\hline & & PZT-5H & PZT-8 & PZT-5A & PMN-30PT & PMN-31PT & PIN-PMN-PT \\
\hline \multirow{3}{*}{$\overline{\overline{m_{1}^{M}}}$} & SS & 0.1804 & 0.1804 & 0.1802 & 0.1794 & 0.1797 & 0.1797 \\
\hline & FS & 0.1804 & 0.1806 & 0.1807 & 0.1803 & 0.1804 & 0.1803 \\
\hline & $\mathrm{L}$ & 0.1797 & 0.1798 & 0.1798 & 0.1796 & 0.1796 & 0.1795 \\
\hline \multirow{3}{*}{$\overline{\overline{m_{2}^{M}}}$} & SS & 0.2628 & 0.2627 & 0.2623 & 0.2599 & 0.2607 & 0.2606 \\
\hline & FS & 0.2627 & 0.2632 & 0.2634 & 0.2624 & 0.2625 & 0.2624 \\
\hline & $\mathrm{L}$ & 0.2606 & 0.2608 & 0.2607 & 0.2599 & 0.2599 & 0.2598 \\
\hline
\end{tabular}

Table 1: Mesh-average of the mean values of the first and second reference dimensionless wave number.

Finally, by the mesh-averaged reference values of total energy error Eq. (38b), the averaged values of the coefficients $A$ and $B$ for the cubic correlation Eq. (34) can be computed,

$$
\overline{e_{T 1,2}^{R M S}}=\left[\bar{A} * e_{h 1,2}+\bar{B}\right] * e_{h 1,2}^{2}
$$

Then the averaged correlation between the total energy error and the percentage of higher order total energy will be yielded

$$
e_{T}^{R M S}=\left(\bar{A} * e_{h}+\bar{B}\right) * e_{h}^{2}, \quad 0<e_{h} \leq e_{h 2}
$$

\section{MODAL ANALYSIS}

As application it is explored the use of the averaged correlation Eq. (40) as a reference to apply the higher order total energy as an indicator of discretization error for the finite element vibration eigenmodes. These ones are the solution of the eigenproblem obtained by considering the homogeneous part of Eq. (1) and assuming stationary waves. In condensed form [10], 


$$
\begin{gathered}
\mathbf{K}^{*} \tilde{\mathbf{x}}_{j}=\omega_{j}^{2} \mathbf{M} \tilde{\mathbf{x}}_{j}, \quad \mathbf{K}^{*}=\mathbf{K}_{u u}+\mathbf{K}_{u \phi} \mathbf{K}_{\phi \phi}^{-1} \mathbf{K}_{u \phi}^{T} \quad(a) \quad \tilde{\phi}_{j}=\mathbf{K}_{\phi \phi}^{-1} \mathbf{K}_{u \phi}^{T} \tilde{\mathbf{x}}_{j} \quad(b) \quad j=1, \ldots, n \\
\tilde{\mathbf{x}}_{i}^{T} \mathbf{K}^{*} \tilde{\mathbf{x}}_{j}=\delta_{i j} \omega_{j}^{2} \quad i, j=1, \ldots, n
\end{gathered}
$$

\begin{tabular}{|c|c|c|c|c|c|c|c|}
\hline & & \multicolumn{3}{|c|}{ HE20 } & \multicolumn{3}{|c|}{ HE20 } \\
\hline & & PZT-5H & PZT-8 & PZT-5A & PMN-30PT & PMN-31PT & PIN-PMN-PT \\
\hline \multirow{3}{*}{$\overline{e_{T 1}^{R M S}}$} & SS & 0.003122 & 0.002918 & 0.002759 & 0.003173 & 0.003013 & 0.002986 \\
\hline & FS & 0.002698 & 0.002818 & 0.003055 & 0.002731 & 0.002780 & 0.002761 \\
\hline & $\mathrm{L}$ & 0.005648 & 0.005287 & 0.005546 & 0.006690 & 0.006288 & 0.006223 \\
\hline \multirow{5}{*}{$\overline{e_{T 2}^{R M S}}$} & SS & 0.013157 & 0.012431 & 0.011570 & 0.012833 & 0.012206 & 0.012081 \\
\hline & FS & 0.011348 & 0.011925 & 0.012891 & 0.011663 & 0.011962 & 0.011782 \\
\hline & $\mathrm{L}$ & 0.029571 & 0.026939 & 0.028789 & 0.038235 & 0.034769 & 0.034268 \\
\hline & & \multicolumn{3}{|c|}{ PE15 } & \multicolumn{3}{|c|}{ PE15 } \\
\hline & & PZT-5H & PZT-8 & PZT-5A & PMN-30PT & PMN-31PT & PIN-PMN-PT \\
\hline \multirow{3}{*}{$\overline{e_{T 1}^{R M S}}$} & SS & 0.005515 & 0.005209 & 0.005389 & 0.006292 & 0.006286 & 0.006180 \\
\hline & FS & 0.005529 & 0.005393 & 0.005588 & 0.005483 & 0.005412 & 0.005505 \\
\hline & $\mathrm{L}$ & 0.007119 & 0.006555 & 0.007139 & 0.008989 & 0.007935 & 0.007664 \\
\hline \multirow{3}{*}{$\overline{e_{T 2}^{R M S}}$} & SS & 0.022301 & 0.021252 & 0.021783 & 0.023798 & 0.024103 & 0.023750 \\
\hline & FS & 0.022770 & 0.022237 & 0.023049 & 0.022635 & 0.022367 & 0.022743 \\
\hline & $\mathrm{L}$ & 0.033449 & 0.029710 & 0.033447 & 0.052871 & 0.043976 & 0.043680 \\
\hline
\end{tabular}

where: $\omega_{j}$ and $\left(\tilde{\mathbf{x}}_{j}, \tilde{\boldsymbol{\phi}}_{j}\right)^{T}$ are the finite element natural frequencies and eigenvectors.

\begin{tabular}{|c|c|c|c|c|c|c|c|}
\hline & & \multicolumn{3}{|c|}{ HE20 } & \multicolumn{3}{|c|}{ HE20 } \\
\hline & & PZT-5H & PZT-8 & PZT-5A & PMN-30PT & PMN-31PT & PIN-PMN-PT \\
\hline \multirow{3}{*}{$\overline{e_{p 2}^{M}}$} & $\mathrm{SS}$ & 1.00392 & 1.00326 & 1.00342 & 1.00752 & 1.00665 & 1.00654 \\
\hline & FS & 1.00356 & 1.00352 & 1.00385 & 1.00321 & 1.00304 & 1.00310 \\
\hline & $\mathrm{L}$ & 1.00194 & 1.00199 & 1.00196 & 1.00168 & 1.00170 & 1.00171 \\
\hline \multirow{5}{*}{$\overline{e_{g n 2}^{M}}$} & SS & 1.01941 & 1.01614 & 1.01692 & 1.03698 & 1.03269 & 1.03219 \\
\hline & FS & 1.01759 & 1.01740 & 1.01902 & 1.01583 & 1.01497 & 1.01525 \\
\hline & $\mathrm{L}$ & 1.00936 & 1.00964 & 1.00947 & 1.00795 & 1.00809 & 1.00815 \\
\hline & & & PE15 & & & PE15 & \\
\hline & & PZT-5H & PZT-8 & PZT-5A & PMN-30PT & PMN-31PT & PIN-PMN-PT \\
\hline \multirow{3}{*}{$\overline{e_{p 2}^{M}}$} & SS & 1.00386 & 1.00330 & 1.00354 & 1.00640 & 1.00568 & 1.00575 \\
\hline & FS & 1.00352 & 1.00339 & 1.00365 & 1.00340 & 1.00322 & 1.00328 \\
\hline & $\mathrm{L}$ & 1.00185 & 1.00191 & 1.00187 & 1.00164 & 1.00167 & 1.00165 \\
\hline \multirow{3}{*}{$\overline{\overline{e_{g n 2}^{M}}}$} & SS & 1.01864 & 1.01596 & 1.01708 & 1.03090 & 1.02748 & 1.02783 \\
\hline & FS & 1.01718 & 1.01653 & 1.01781 & 1.01652 & 1.01561 & 1.01588 \\
\hline & $\mathrm{L}$ & 1.00897 & 1.00914 & 1.00891 & 1.00673 & 1.00739 & 1.00717 \\
\hline
\end{tabular}

Table 2: Mesh-average of the rms values of the first and second reference percentage of total energy error.

Table 3: Second mesh-averaged reference values of the indicators of numerical dispersion.

Eq. (41a) and Eq. (41b) are solved by LAPACK routines [25]. By considering the relation of K-orthogonality Eq. (42) for the condensed eigenproblem Eq. (40a), and taking into account Eq. (41b) and Eq. (7), it can be yielded the following expression for the modal total energy

$$
\frac{1}{4} \tilde{\mathbf{x}}_{j}^{T} \mathbf{K}_{u u} \tilde{\mathbf{x}}_{j}+\frac{1}{4} \tilde{\boldsymbol{\phi}}_{j}^{T} \mathbf{K}_{\phi \phi} \tilde{\boldsymbol{\phi}}_{j}=\frac{1}{4} \omega_{j}^{2} \rightarrow \bar{E}_{j}=\frac{1}{4} \omega_{j}^{2}, \quad j=1, \ldots, n
$$


The error for the modal total energy computed with the discretized piezoelectric domain is estimated by a reference model that will be obtained by dividing each element of the actual mesh into eight elements. The modal total energy computed with the actual model and the one computed with the reference model will be compared for each eigenmode by

$$
T E E=\left(\bar{E} / \bar{E}^{R E F}\right)-1, \quad T E E=\left(\omega / \omega^{R E F}\right)^{2}-1
$$

The modal total energy error Eq. (44) will be compared with a proposed total energy error indicator which is computed either by averaging the correlation Eq. (40) for the slow and fast quasi-shear waves, or by the correlation Eq. (40) for the quasi-longitudinal waves,

$$
\operatorname{TEES}(P H E)=\frac{1}{2}\left(e_{T, S S}^{R M S}+e_{T, F S}^{R M S}\right) \quad(a) \quad T E E L(P H E)=e_{T, L}^{R M S} \quad(b), \quad 0<P H E \leq e_{h 2}
$$

where $P H E$ is the percentage of higher order total energy computed with the actual model.

From Table 2, the following inequality is clearly fulfilled,

$$
\text { TEES }(P H E)<T E E L(P H E)
$$

Finally, by taking into account Eq. (7), the percentage of modal electric energy will be also computed with the actual model,

$$
P E E=\bar{E}^{E} /\left(\bar{E}^{M}+\bar{E}^{E}\right)
$$

It must be remarked that, for plane bulk acoustic waves, Eq. (47) would be an accepted definition of electromechanical coupling coefficient from energy consideration [26].

\subsection{Numerical research}

Two test problems discretized by the HE20 element have been analyzed, Fig. 4: a piezoelectric cantilever bimorph connected in parallel configuration made of PZT-5A $(\mathrm{L}=16$ $\mathrm{mm}, \mathrm{b}=3 \mathrm{~mm}, \mathrm{t}=2 \mathrm{~mm}$ ) $[27,28]$, and a piezoelectric cube laterally clamped made of PZT-5H or, alternatively, PMN-30PT single crystal $(\mathrm{L}=1 \mathrm{~mm})$ [29]. A piezoelectric disk laterally clamped, discretized by the PE15 element, is also analyzed, Fig. $5(\mathrm{R}=12 \mathrm{~mm}, \mathrm{t}=6 \mathrm{~mm})$. The piezoelectric disk is made of PZT-8 or, alternatively, PIN-PMN-PT single crystal. In each test problem the electrodes are shorted and their mechanical effects are neglected.

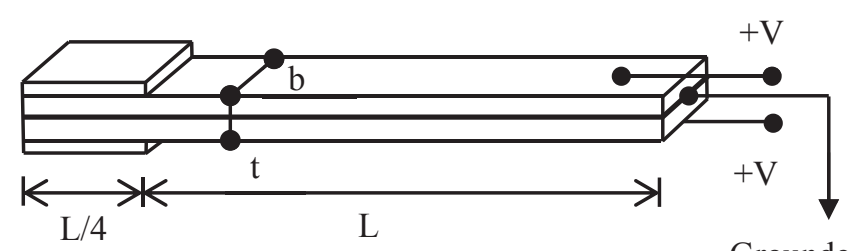

Grounded

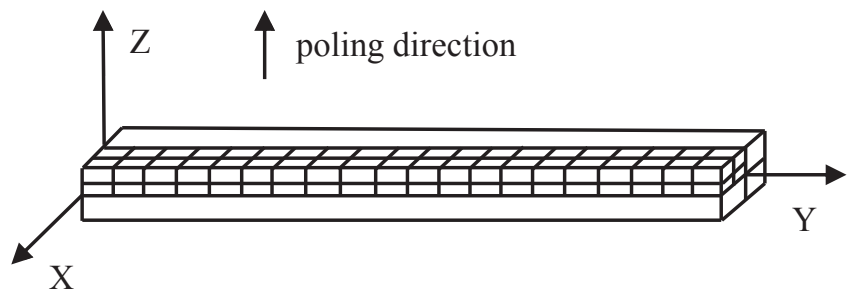

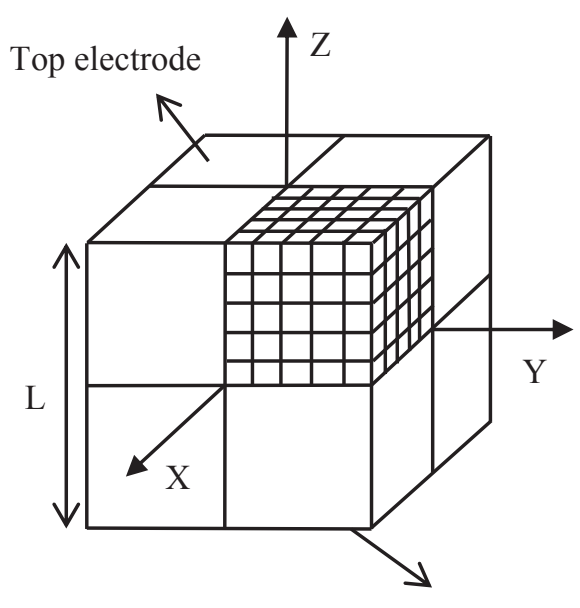

Bottom electrode

Figure 4: Piezoelectric cantilever bimorph and cube laterally clamped discretized by the HE20 element. 
Since the piezoelectric bimorph has two planes of symmetry, which are $X Y$ and $Y Z$, the modes can be computed by idealizing one quarter of it and applying four combinations of boundary conditions on the two planes for symmetric motion (S) and for antisymmetric motion (A), see details in reference [30]. In this test problem, the swaying modes in the Z-direction (AS) and the torsion modes about the Y-axis (AA) have been computed, Table 4.
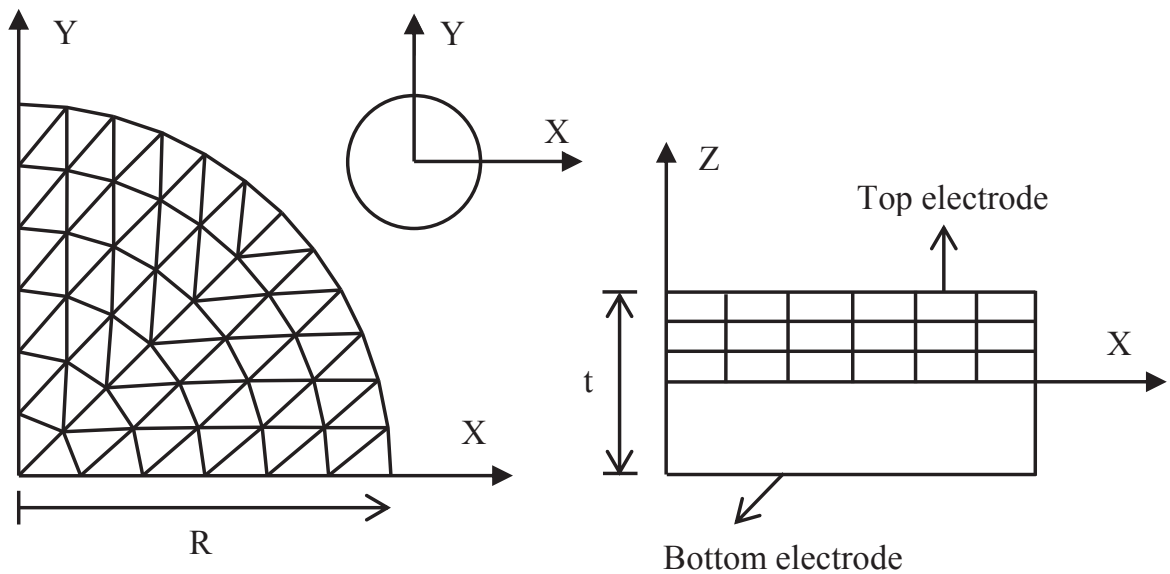

Figure 5: Piezoelectric disk laterally clamped discretized by the PE15 element.

Similarly, since both the piezoelectric cube and the piezoelectric disk have three planes of symmetry, which are $Y Z, X Z$ and $X Y$, the modes can be computed by idealizing one eighth of them, and applying six combinations of boundary conditions on the three planes for symmetric motion (S) and for antisymmetric motion (A). Also see details in reference [30]. In both test problems, the antisymmetric and symmetric longitudinal modes in the Z-direction (SSA and SSS), the antisymmetric and symmetric swaying modes in the Y-direction (SAA and SAS), and the antisymmetric and symmetric torsion modes about the Z-axis (AAA and AAS) have been computed. The results are displayed in Tables 5-16.

For each test problem it is selected the set of consecutive eigenmodes for which the following condition is fulfilled: $0<P H E \leq e_{h 2}$ (see Eq. (35)).

\begin{tabular}{ccrccccc}
\hline \#MODE & & \multicolumn{1}{c}{ FR $(\mathrm{Hz})$} & PHE & TEE & TEES & PHE REF & PEE \\
\hline 1 & AS & 3631.35 & 0.088188 & 0.005768 & 0.002247 & 0.026710 & 0.027781 \\
2 & AS & 21217.95 & 0.092730 & 0.005303 & 0.002490 & 0.027706 & 0.031491 \\
3 & AA & 22773.80 & 0.139349 & 0.002560 & 0.005760 & 0.039546 & 0.006289 \\
4 & AS & 54358.47 & 0.103447 & 0.004919 & 0.003116 & 0.030374 & 0.038010 \\
5 & AA & 68479.08 & 0.146347 & 0.002731 & 0.006376 & 0.041704 & 0.008586 \\
6 & AS & 96273.68 & 0.120632 & 0.004907 & 0.004276 & 0.034929 & 0.046683 \\
7 & AA & 114645.43 & 0.160009 & 0.003132 & 0.007674 & 0.045951 & 0.012958 \\
8 & AS & 143956.67 & 0.143424 & 0.005613 & 0.006114 & 0.041300 & 0.056198 \\
8 & AA & 161540.12 & 0.179748 & 0.003882 & 0.009780 & 0.052171 & 0.019010 \\
10 & AS & 195356.94 & 0.170984 & 0.007262 & 0.008811 & 0.049380 & 0.065731 \\
11 & AA & 209366.65 & 0.204825 & 0.005154 & 0.012858 & 0.026253 & 0.060229 \\
12 & AS & 249168.34 & 0.201768 & 0.009994 & 0.012459 & 0.074345 & 0.058857 \\
\hline
\end{tabular}

Table 4: PZT-5A cantilever bimorph. Proposed total energy error indicator TEES versus total energy error computed by mesh halving TEE. 
Francisco J. Brito Castro

\begin{tabular}{cccccccc}
\hline \#MODE & & FR $(\mathrm{Hz})$ & PHE & TEE & TEES & PHE REF & PEE \\
\hline 1 & A & 1494798.75 & 0.044947 & 0.001012 & 0.000571 & 0.012649 & 0.378348 \\
2 & S & 2253607.19 & 0.054692 & 0.000674 & 0.000850 & 0.014552 & 0.257970 \\
3 & A & 2911828.99 & 0.103631 & 0.002572 & 0.003131 & 0.030457 & 0.160737 \\
4 & S & 2925280.47 & 0.104319 & 0.003437 & 0.003174 & 0.031274 & 0.075176 \\
5 & A & 2949444.83 & 0.103972 & 0.003440 & 0.003152 & 0.031517 & 0.070138 \\
6 & S & 3219709.13 & 0.111434 & 0.002536 & 0.003635 & 0.030942 & 0.194094 \\
7 & A & 3269260.33 & 0.132101 & 0.003350 & 0.005164 & 0.037684 & 0.242467 \\
8 & S & 3293030.56 & 0.122009 & 0.004621 & 0.004382 & 0.037425 & 0.138397 \\
9 & S & 3343606.11 & 0.123991 & 0.003496 & 0.004530 & 0.035570 & 0.043260 \\
10 & A & 3407876.53 & 0.106406 & 0.002466 & 0.003306 & 0.029866 & 0.345914 \\
11 & S & 3470792.89 & 0.108399 & 0.002632 & 0.003434 & 0.030465 & 0.269794 \\
12 & A & 3546463.33 & 0.102105 & 0.001708 & 0.003037 & 0.027275 & 0.170078 \\
13 & A & 3727337.34 & 0.127277 & 0.003860 & 0.004782 & 0.035938 & 0.082845 \\
14 & A & 3730441.23 & 0.120325 & 0.004203 & 0.004258 & 0.034218 & 0.098911 \\
15 & S & 3785874.14 & 0.130364 & 0.002186 & 0.005024 & 0.035527 & 0.050604 \\
16 & S & 4064314.52 & 0.163973 & 0.005918 & 0.008088 & 0.050438 & 0.115876 \\
17 & A & 4115269.62 & 0.191198 & 0.005907 & 0.011148 & 0.059473 & 0.164358 \\
18 & S & 4218250.54 & 0.142201 & 0.004665 & 0.006015 & 0.039927 & 0.084108 \\
19 & A & 4223091.77 & 0.165183 & 0.002378 & 0.008212 & 0.045304 & 0.032168 \\
20 & S & 4354513.22 & 0.178546 & 0.006200 & 0.009660 & 0.051740 & 0.173579 \\
21 & S & 4440349.35 & 0.154910 & 0.005003 & 0.007185 & 0.044617 & 0.090900 \\
22 & S & 4475618.52 & 0.170321 & 0.006235 & 0.008754 & 0.047812 & 0.066334 \\
23 & A & 4481882.47 & 0.171154 & 0.006331 & 0.008844 & 0.050257 & 0.167460 \\
24 & A & 4530610.59 & 0.166572 & 0.005410 & 0.008357 & 0.050164 & 0.293279 \\
25 & S & 4569360.37 & 0.204520 & 0.008802 & 0.012841 & 0.059976 & 0.112327 \\
26 & A & 4691002.54 & 0.200371 & 0.009031 & 0.012300 & 0.058179 & 0.099302 \\
27 & A & 4755510.15 & 0.192601 & 0.005597 & 0.011321 & 0.055896 & 0.129024 \\
28 & S & 4796773.25 & 0.170910 & 0.007932 & 0.008817 & 0.052541 & 0.097511 \\
\hline
\end{tabular}

Table 5: PZT-5H cube laterally clamped. Proposed total energy error indicator TEES versus total energy error computed by mesh halving TEE. Modes SS (S) and SS (A).

\begin{tabular}{cccccccc}
\hline \#MODE & & FR $(\mathrm{Hz})$ & PHE & TEE & TEEL & PHE REF & PEE \\
\hline 1 & A & 1955083.91 & 0.047784 & 0.004777 & 0.001186 & 0.014847 & 0.285874 \\
2 & S & 2070385.14 & 0.105834 & 0.009124 & 0.007681 & 0.028318 & 0.006049 \\
3 & S & 2347003.92 & 0.062630 & 0.002363 & 0.002204 & 0.016782 & 0.332819 \\
4 & A & 2368802.89 & 0.113605 & 0.008700 & 0.009138 & 0.032137 & 0.029402 \\
5 & A & 2594239.22 & 0.119986 & 0.009462 & 0.010457 & 0.037010 & 0.229177 \\
6 & S & 2904544.18 & 0.144480 & 0.013562 & 0.016629 & 0.043907 & 0.180060 \\
7 & S & 2947810.19 & 0.169046 & 0.026569 & 0.024778 & 0.055882 & 0.098489 \\
\hline
\end{tabular}

Table 6: PMN-30PT cube laterally clamped. Proposed total energy error indicator TEEL versus total energy error computed by mesh halving TEE. Modes SS (S) and SS (A).

From Tables 4-16 it is deduced that the percentage of higher order total energy decreases as the mesh is refined. Then, it is verified that this energy component behaves as a modal error indicator, which is in accordance with the numerical dispersion analysis.

From Tables 4, 5, 7, 9, 11, 13 and 15 it can be deduced that, for the solids made of PZT piezoceramics, the proposed total energy error indicator based on S-waves Eq. (45a) and the 
total energy error computed by mesh halving Eq. (44) generally exhibit a similar evolution pattern as the modal order increases. Also, the proposed total energy error indicator Eq. (45a) generally overestimates the total energy error computed by mesh halving Eq. (44). Clearly, by the proposed total energy error indicator based on S-waves Eq. (45a), the accuracy of the finite element eigenmodes could be confidently verified in order to select a cutoff modal order given an upper bound for the total energy error close to one per cent.

The above behavior for the solids made of PZT piezoceramics could be expected based on the heuristic that, given the natural frequency, both the percentage of higher order total energy and the spatial discretization error should be mainly influenced by the error induced by the slowest quasi-shear waves which have the shortest wavelengths.

\begin{tabular}{|c|c|c|c|c|c|c|c|}
\hline \#MODE & & FR (Hz) & PHE & TEE & TEES & PHE REF & PEE \\
\hline 1 & $\mathrm{~S}$ & 1940056.24 & 0.037057 & 0.001392 & 0.000386 & 0.011346 & 0.131090 \\
\hline 2 & A & 2089555.22 & 0.037879 & 0.000869 & 0.000404 & 0.010915 & 0.195819 \\
\hline 3 & A & 2434954.09 & 0.079598 & 0.002009 & 0.001824 & 0.023051 & 0.374945 \\
\hline 4 & $\mathrm{~S}$ & 2487876.85 & 0.069741 & 0.001248 & 0.001393 & 0.019433 & 0.238560 \\
\hline 5 & $\mathrm{~S}$ & 2828993.11 & 0.080055 & 0.001657 & 0.001845 & 0.021692 & 0.044895 \\
\hline 6 & A & 2982767.69 & 0.088200 & 0.001334 & 0.002250 & 0.023570 & 0.057256 \\
\hline 7 & $\mathrm{~S}$ & 3140640.11 & 0.090835 & 0.001926 & 0.002389 & 0.025225 & 0.186659 \\
\hline 8 & A & 3297569.35 & 0.102153 & 0.002622 & 0.003040 & 0.029069 & 0.188117 \\
\hline 9 & $\mathrm{~S}$ & 3421891.52 & 0.123931 & 0.002632 & 0.004526 & 0.035635 & 0.075841 \\
\hline 10 & A & 3467280.17 & 0.139115 & 0.003948 & 0.005748 & 0.041684 & 0.124472 \\
\hline 11 & $\mathrm{~S}$ & 3612688.32 & 0.126168 & 0.003720 & 0.004696 & 0.036407 & 0.106855 \\
\hline 12 & A & 3775918.50 & 0.141560 & 0.003737 & 0.005959 & 0.040244 & 0.174574 \\
\hline 13 & $\mathrm{~S}$ & 3780264.69 & 0.128603 & 0.002931 & 0.004885 & 0.036160 & 0.190129 \\
\hline 14 & $\mathrm{~S}$ & 3901737.88 & 0.164548 & 0.005014 & 0.008147 & 0.047153 & 0.089534 \\
\hline 15 & A & 3904222.86 & 0.151162 & 0.003813 & 0.006828 & 0.044073 & 0.255638 \\
\hline 16 & A & 4005566.29 & 0.159113 & 0.003850 & 0.007596 & 0.045297 & 0.124791 \\
\hline 17 & $\mathrm{~S}$ & 4136640.68 & 0.181068 & 0.006140 & 0.009948 & 0.053140 & 0.203126 \\
\hline 18 & A & 4178781.08 & 0.155419 & 0.003933 & 0.007234 & 0.044197 & 0.193035 \\
\hline 19 & A & 4228182.19 & 0.167523 & 0.004781 & 0.008457 & 0.047798 & 0.067265 \\
\hline 20 & $\mathrm{~S}$ & 4249965.73 & 0.187775 & 0.007818 & 0.010734 & 0.057300 & 0.198316 \\
\hline 21 & $\mathrm{~S}$ & 4364136.58 & 0.167402 & 0.005350 & 0.008444 & 0.046784 & 0.079209 \\
\hline 22 & $\mathrm{~S}$ & 4501655.31 & 0.182042 & 0.006794 & 0.010060 & 0.050711 & 0.066730 \\
\hline 23 & A & 4540145.24 & 0.163551 & 0.005669 & 0.008044 & 0.047357 & 0.312658 \\
\hline 24 & A & 4620112.25 & 0.190505 & 0.007098 & 0.011064 & 0.052324 & 0.055107 \\
\hline 25 & $\mathrm{~S}$ & 4633649.48 & 0.179765 & 0.005468 & 0.009798 & 0.050850 & 0.123484 \\
\hline 26 & $\mathrm{~S}$ & 4688950.56 & 0.174831 & 0.005880 & 0.009245 & 0.049616 & 0.161655 \\
\hline 27 & A & 4758178.95 & 0.185452 & 0.006966 & 0.010458 & 0.054770 & 0.122037 \\
\hline 28 & $\mathrm{~S}$ & 4795991.67 & 0.195165 & 0.005529 & 0.011639 & 0.056789 & 0.092402 \\
\hline 29 & A & 4858675.43 & 0.203334 & 0.007203 & 0.012686 & 0.060966 & 0.174814 \\
\hline
\end{tabular}

Table 7: PZT-5H cube laterally clamped. Proposed total energy error indicator TEES versus total energy error computed by mesh halving TEE. Modes SA (S) and SA (A).

From Tables 5-16, it can be deduced that the modal total energy errors computed by mesh halving for the solids made of PMN-PT $<001>$ poled single crystals are significantly greater than the ones computed for the solids made of PZT. This behavior is similar to the dilatational locking phenomenon that is most commonly associated with nearly incompressible isotropic materials, i.e., with those for which the Poisson's ratio approaches one-half very closely [13]. 
From Tables $6,8,10,12,14$ and 16 it can be deduced that, for the solids made of PMN-PT single crystals, only the proposed total energy error indicator based on L-waves Eq. (45b) overestimates for some eigenmodes the total energy error computed by mesh halving Eq. (44); however, by the proposed total energy error indicator based on L-waves Eq. (45b), an upper bound for the total energy error generally can be satisfactorily estimated for the set of eigenmodes selected. Clearly, for the PMN-PT solids, the modal total energy error should be mainly influenced by the error induced by the quasi-longitudinal waves. Both for PMN-30PT cube and for the PIN-PMN-PT disk laterally clamped, the total energy errors computed by mesh halving, for the eigenmodes selected, generally can be accepted for all practical purposes from the engineering point of view.

\begin{tabular}{cccccccc}
\hline \#MODE & & FR $(\mathrm{Hz})$ & PHE & TEE & TEEL & PHE REF & PEE \\
\hline 1 & S & 1831206.39 & 0.056366 & 0.007051 & 0.001728 & 0.016903 & 0.079782 \\
2 & A & 2215758.70 & 0.046708 & 0.004171 & 0.001126 & 0.014119 & 0.221063 \\
3 & S & 2405588.22 & 0.110802 & 0.012973 & 0.008594 & 0.032335 & 0.076049 \\
4 & A & 2664535.32 & 0.117330 & 0.010324 & 0.009894 & 0.034096 & 0.103798 \\
5 & S & 2794592.00 & 0.126270 & 0.014561 & 0.011868 & 0.035678 & 0.121444 \\
6 & S & 2949954.65 & 0.090059 & 0.009531 & 0.005195 & 0.025816 & 0.179932 \\
7 & A & 3046735.64 & 0.097286 & 0.005731 & 0.006258 & 0.028028 & 0.330363 \\
8 & A & 3090706.07 & 0.150862 & 0.016097 & 0.018547 & 0.042078 & 0.033922 \\
\hline
\end{tabular}

Table 8: PMN-30PT cube laterally clamped. Proposed total energy error indicator TEEL versus total energy error computed by mesh halving TEE. Modes SA (S) and SA (A).

\begin{tabular}{cccccccc}
\hline \#MODE & & FR $(\mathrm{Hz})$ & PHE & TEE & TEES & PHE REF & PEE \\
\hline 1 & S & 2024859.86 & 0.044406 & 0.000407 & 0.000557 & 0.011471 & 0.000216 \\
2 & A & 2303313.34 & 0.052876 & 0.000465 & 0.000793 & 0.014074 & 0.082242 \\
3 & S & 2724869.55 & 0.070839 & 0.002311 & 0.001438 & 0.020973 & 0.147724 \\
4 & A & 2809855.32 & 0.062111 & 0.001722 & 0.001100 & 0.018002 & 0.144506 \\
5 & S & 2937759.52 & 0.082856 & 0.001065 & 0.001980 & 0.022996 & 0.152725 \\
6 & S & 3039991.26 & 0.090128 & 0.001847 & 0.002352 & 0.025490 & 0.190031 \\
7 & A & 3050295.13 & 0.112990 & 0.002373 & 0.003741 & 0.032682 & 0.375057 \\
8 & S & 3549794.00 & 0.122028 & 0.003297 & 0.004383 & 0.034450 & 0.164487 \\
9 & A & 3617277.27 & 0.125511 & 0.001897 & 0.004646 & 0.034861 & 0.136241 \\
10 & S & 3635257.08 & 0.129175 & 0.003529 & 0.004930 & 0.034367 & 0.000358 \\
11 & A & 3668364.29 & 0.115199 & 0.002482 & 0.003893 & 0.032078 & 0.189753 \\
12 & A & 3765104.77 & 0.136739 & 0.003342 & 0.005546 & 0.036684 & 0.015491 \\
13 & A & 3887782.42 & 0.136244 & 0.003633 & 0.005505 & 0.037601 & 0.063732 \\
14 & S & 3893669.38 & 0.122578 & 0.003667 & 0.004424 & 0.033558 & 0.054943 \\
15 & S & 4121863.35 & 0.159137 & 0.003041 & 0.007599 & 0.043516 & 0.038288 \\
16 & S & 4262559.26 & 0.157842 & 0.003452 & 0.007471 & 0.044082 & 0.125908 \\
17 & A & 4269201.31 & 0.175189 & 0.004846 & 0.009284 & 0.052109 & 0.234513 \\
18 & S & 4270499.91 & 0.184011 & 0.007730 & 0.010289 & 0.053881 & 0.049026 \\
19 & A & 4305152.76 & 0.191207 & 0.007863 & 0.011150 & 0.057651 & 0.078252 \\
20 & S & 4430778.46 & 0.185779 & 0.004550 & 0.010497 & 0.052688 & 0.141208 \\
21 & S & 4452728.08 & 0.186311 & 0.005351 & 0.010560 & 0.053636 & 0.169705 \\
\hline
\end{tabular}

Table 9: PZT-5H cube laterally clamped. Proposed total energy error indicator TEES versus total energy error computed by mesh halving TEE. Modes AA (S) and AA (A). 
Francisco J. Brito Castro

\begin{tabular}{cccccccc}
\hline \#MODE & & FR $(\mathrm{Hz})$ & PHE & TEE & TEEL & PHE REF & PEE \\
\hline 1 & S & 1696277.98 & 0.043646 & 0.001614 & 0.000966 & 0.011304 & 0.000239 \\
2 & A & 2288255.39 & 0.051260 & 0.001024 & 0.001390 & 0.013540 & 0.118416 \\
3 & S & 2600469.55 & 0.187436 & 0.027305 & 0.032316 & 0.049783 & 0.002476 \\
4 & S & 2835507.34 & 0.091854 & 0.011700 & 0.005447 & 0.027974 & 0.036460 \\
5 & A & 2943475.43 & 0.191199 & 0.021385 & 0.034021 & 0.052110 & 0.048119 \\
6 & A & 3031360.11 & 0.092986 & 0.011084 & 0.005610 & 0.029612 & 0.107099 \\
7 & S & 3437664.02 & 0.173580 & 0.041123 & 0.026517 & 0.067656 & 0.032985 \\
8 & S & 3474206.51 & 0.106232 & 0.005290 & 0.007752 & 0.023311 & 0.134139 \\
9 & S & 3491292.14 & 0.113828 & 0.005874 & 0.009182 & 0.032752 & 0.246306 \\
\hline
\end{tabular}

Table 10: PMN-30PT cube laterally clamped. Proposed total energy error indicator TEEL versus total energy error computed by mesh halving TEE. Modes AA (S) and AA (A).

\begin{tabular}{cccccccc}
\hline \#MODE & & FR $(\mathrm{Hz})$ & PHE & TEE & TEES & PHE REF & PEE \\
\hline 1 & A & 51541.31 & 0.048021 & 0.002249 & 0.002153 & 0.013658 & 0.177725 \\
2 & A & 124164.61 & 0.068596 & 0.003176 & 0.004425 & 0.019245 & 0.209649 \\
3 & A & 136836.01 & 0.079258 & 0.003836 & 0.005929 & 0.022247 & 0.215999 \\
4 & S & 150662.77 & 0.030442 & 0.001521 & 0.000860 & 0.008708 & 0.053842 \\
5 & S & 189790.61 & 0.030784 & 0.001357 & 0.000880 & 0.008937 & 0.116753 \\
6 & S & 194972.83 & 0.045667 & 0.002401 & 0.001946 & 0.012235 & 0.029768 \\
7 & A & 198719.09 & 0.094287 & 0.005113 & 0.008433 & 0.026825 & 0.221408 \\
8 & A & 217589.92 & 0.072451 & 0.004112 & 0.004942 & 0.020729 & 0.105793 \\
9 & S & 229412.09 & 0.064027 & 0.004506 & 0.003849 & 0.017764 & 0.023877 \\
10 & A & 230820.02 & 0.116057 & 0.008231 & 0.012870 & 0.033558 & 0.201034 \\
11 & A & 231941.28 & 0.099038 & 0.005996 & 0.009319 & 0.027954 & 0.123675 \\
12 & A & 251548.75 & 0.069560 & 0.003639 & 0.004551 & 0.019548 & 0.035352 \\
13 & A & 257018.78 & 0.070986 & 0.001782 & 0.004742 & 0.018867 & 0.003714 \\
14 & A & 272961.42 & 0.134343 & 0.009464 & 0.017351 & 0.039191 & 0.228955 \\
15 & S & 273635.96 & 0.101763 & 0.006729 & 0.009848 & 0.027661 & 0.070426 \\
16 & S & 277908.60 & 0.089192 & 0.006175 & 0.007533 & 0.024691 & 0.090064 \\
17 & A & 281742.73 & 0.080465 & 0.004068 & 0.006113 & 0.022439 & 0.015318 \\
18 & S & 287413.21 & 0.110412 & 0.003803 & 0.011627 & 0.030509 & 0.175302 \\
19 & S & 287709.83 & 0.105576 & 0.006213 & 0.010613 & 0.029050 & 0.105030 \\
20 & S & 298220.48 & 0.104759 & 0.010929 & 0.010447 & 0.029507 & 0.021874 \\
\hline
\end{tabular}

Table 11: PZT-8 disk laterally clamped. Proposed total energy error indicator TEES versus total energy error computed by mesh halving TEE. Modes SS (S) and SS (A).

\begin{tabular}{ccrccccc}
\hline \#MODE & & FR $(\mathrm{Hz})$ & PHE & TEE & TEEL & PHE REF & PEE \\
\hline 1 & A & 50070.97 & 0.061813 & 0.012118 & 0.004817 & 0.020158 & 0.282503 \\
2 & S & 92783.92 & 0.043018 & 0.003907 & 0.002065 & 0.011601 & 0.116988 \\
3 & S & 122410.01 & 0.064886 & 0.011353 & 0.005408 & 0.019192 & 0.183122 \\
4 & A & 123322.12 & 0.076182 & 0.013804 & 0.007960 & 0.024150 & 0.262586 \\
5 & A & 138967.48 & 0.086335 & 0.014515 & 0.010808 & 0.026849 & 0.245642 \\
6 & S & 146426.59 & 0.050728 & 0.005403 & 0.003024 & 0.014895 & 0.390335 \\
7 & S & 147337.01 & 0.112765 & 0.022865 & 0.021031 & 0.030776 & 0.057746 \\
8 & S & 154795.39 & 0.081233 & 0.009450 & 0.009308 & 0.022474 & 0.086916 \\
\hline
\end{tabular}

Table 12: PIN-PMN-PT disk laterally clamped. Proposed total energy error indicator TEEL versus total energy error computed by mesh halving TEE. Modes SS (S) and SS (A). 
Francisco J. Brito Castro

\begin{tabular}{cccccccc}
\hline \#MODE & & FR $(\mathrm{Hz})$ & PHE & TEE & TEES & PHE REF & PEE \\
\hline 1 & $\mathrm{~A}$ & 87719.21 & 0.058070 & 0.002506 & 0.003160 & 0.016293 & 0.201956 \\
2 & $\mathrm{~S}$ & 99192.21 & 0.011349 & 0.000872 & 0.000119 & 0.003663 & 0.084222 \\
3 & $\mathrm{~S}$ & 150752.21 & 0.026183 & 0.001172 & 0.000635 & 0.006924 & 0.009380 \\
4 & $\mathrm{~A}$ & 161484.50 & 0.081146 & 0.004074 & 0.006218 & 0.022875 & 0.217459 \\
5 & $\mathrm{~A}$ & 181107.96 & 0.096651 & 0.005297 & 0.008868 & 0.027251 & 0.225069 \\
6 & $\mathrm{~S}$ & 192771.32 & 0.046451 & 0.002909 & 0.002014 & 0.013028 & 0.035684 \\
7 & $\mathrm{~A}$ & 205686.40 & 0.044962 & 0.001804 & 0.001886 & 0.012630 & 0.076821 \\
8 & $\mathrm{~A}$ & 232909.21 & 0.060667 & 0.001457 & 0.003452 & 0.016920 & 0.054281 \\
9 & $\mathrm{~A}$ & 236444.46 & 0.110036 & 0.006662 & 0.011546 & 0.030906 & 0.217513 \\
10 & $\mathrm{~S}$ & 237038.78 & 0.075228 & 0.004841 & 0.005334 & 0.020399 & 0.021474 \\
11 & $\mathrm{~S}$ & 239632.74 & 0.063123 & 0.004437 & 0.003740 & 0.017414 & 0.059033 \\
12 & $\mathrm{~S}$ & 251119.89 & 0.062182 & 0.002908 & 0.003628 & 0.017460 & 0.138893 \\
13 & $\mathrm{~A}$ & 253819.79 & 0.071411 & 0.003699 & 0.004800 & 0.020122 & 0.061200 \\
14 & $\mathrm{~S}$ & 264704.16 & 0.084074 & 0.007583 & 0.006682 & 0.023419 & 0.021252 \\
15 & $\mathrm{~A}$ & 270231.84 & 0.138254 & 0.010584 & 0.018400 & 0.040243 & 0.187533 \\
\hline
\end{tabular}

Table 13: PZT-8 disk laterally clamped. Proposed total energy error indicator TEES versus total energy error computed by mesh halving TEE. Modes SA (S) and SA (A).

\begin{tabular}{cccccccc}
\hline \#MODE & & FR $(\mathrm{Hz})$ & PHE & TEE & TEEL & PHE REF & PEE \\
\hline 1 & $\mathrm{~S}$ & 76742.94 & 0.021142 & 0.003894 & 0.000423 & 0.006963 & 0.223332 \\
2 & $\mathrm{~A}$ & 88923.44 & 0.071038 & 0.013679 & 0.006721 & 0.023099 & 0.245894 \\
3 & $\mathrm{~S}$ & 97133.76 & 0.035209 & 0.004302 & 0.001308 & 0.009999 & 0.093410 \\
4 & $\mathrm{~S}$ & 125002.91 & 0.066811 & 0.008671 & 0.005800 & 0.018029 & 0.027904 \\
5 & $\mathrm{~S}$ & 145553.70 & 0.087838 & 0.014946 & 0.011277 & 0.024319 & 0.073728 \\
6 & $\mathrm{~A}$ & 163712.89 & 0.079371 & 0.012223 & 0.008796 & 0.024630 & 0.236595 \\
7 & $\mathrm{~S}$ & 167542.09 & 0.138053 & 0.030572 & 0.035242 & 0.039285 & 0.027827 \\
8 & $\mathrm{~S}$ & 172478.18 & 0.092090 & 0.016765 & 0.012673 & 0.026622 & 0.172292 \\
9 & $\mathrm{~A}$ & 185128.17 & 0.091919 & 0.014160 & 0.012615 & 0.028045 & 0.226468 \\
10 & $\mathrm{~S}$ & 188251.10 & 0.091829 & 0.012304 & 0.012584 & 0.025898 & 0.216315 \\
11 & $\mathrm{~S}$ & 193595.02 & 0.090701 & 0.012885 & 0.012206 & 0.026752 & 0.294166 \\
\hline
\end{tabular}

Table 14: PIN-PMN-PT disk laterally clamped. Proposed total energy error indicator TEEL versus total energy error computed by mesh halving TEE. Modes SA (S) and SA (A).

\section{CONCLUSIONS}

For piezoelectric media discretized either by the energy-orthogonal twenty-node hexahedral finite element or by the energy-orthogonal fifteen-node pentahedral finite element, by a dispersion analysis of plane harmonic bulk waves, an averaged correlation between the percentage of higher order total energy and the total energy error is yielded in each case both for the quasi-longitudinal waves and the quasi-shear waves. The use of such correlations as reference to apply the higher order total energy as an error indicator for the finite element vibration eigenmodes in piezoelectric solids is explored. As noteworthy conclusions:

- For PZT piezoceramics discretized by regular meshes the numerical research reveals that, by the correlation based on quasi-shear waves, the accuracy of the finite element eigenmodes can be confidently verified in order to select a cutoff modal order given an upper bound for the total energy error close to one per cent. 
- For PMN-PT $<001>$ poled single crystals a phenomenon similar to the dilatational locking could affect the accuracy of the eigenmodes computed. In this case, the correlation based on the quasi-longitudinal waves will be more accurate than the one based on the quasi-shear waves. An upper bound for the total energy error generally can be satisfactorily estimated for the set of eigenmodes selected.

\begin{tabular}{cccccccc}
\hline \#MODE & & FR $(\mathrm{Hz})$ & PHE & TEE & TEES & PHE REF & PEE \\
\hline 1 & S & 107006.40 & 0.009654 & 0.000537 & 0.000086 & 0.002590 & 0.000000 \\
2 & A & 124403.79 & 0.068477 & 0.003158 & 0.004409 & 0.019188 & 0.213427 \\
3 & S & 151530.13 & 0.029858 & 0.001511 & 0.000827 & 0.008527 & 0.060573 \\
4 & S & 195671.83 & 0.045609 & 0.002416 & 0.001941 & 0.012245 & 0.035823 \\
5 & S & 196124.61 & 0.052478 & 0.002468 & 0.002575 & 0.013839 & 0.000000 \\
6 & A & 198897.48 & 0.097682 & 0.005574 & 0.009061 & 0.027787 & 0.218012 \\
7 & A & 210858.61 & 0.043840 & 0.000851 & 0.001792 & 0.011718 & 0.084750 \\
8 & A & 222906.51 & 0.099339 & 0.006712 & 0.009377 & 0.028718 & 0.208479 \\
9 & S & 230153.98 & 0.065812 & 0.005252 & 0.004069 & 0.018289 & 0.026005 \\
10 & A & 235937.00 & 0.076905 & 0.003900 & 0.005577 & 0.021555 & 0.080925 \\
11 & A & 267146.81 & 0.081979 & 0.001640 & 0.006348 & 0.021983 & 0.051653 \\
12 & A & 268071.13 & 0.082903 & 0.003441 & 0.006494 & 0.023966 & 0.089605 \\
13 & A & 274323.99 & 0.125060 & 0.008319 & 0.014990 & 0.035278 & 0.222572 \\
14 & S & 275419.64 & 0.103464 & 0.007639 & 0.010186 & 0.028167 & 0.058666 \\
15 & S & 280164.97 & 0.084483 & 0.006603 & 0.006748 & 0.023722 & 0.102002 \\
16 & S & 285448.64 & 0.117184 & 0.009301 & 0.013127 & 0.032024 & 0.000010 \\
17 & A & 285499.46 & 0.084125 & 0.004895 & 0.006690 & 0.023488 & 0.039281 \\
18 & S & 290762.64 & 0.100089 & 0.005552 & 0.009521 & 0.027868 & 0.147950 \\
19 & S & 298812.82 & 0.103531 & 0.010995 & 0.010199 & 0.029058 & 0.021948 \\
\hline
\end{tabular}

Table 15: PZT-8 disk laterally clamped. Proposed total energy error indicator TEES versus total energy error computed by mesh halving TEE. Modes AA (S) and AA (A).

\begin{tabular}{cccccccc}
\hline \#MODE & & FR $(\mathrm{Hz})$ & PHE & TEE & TEEL & PHE REF & PEE \\
\hline 1 & $\mathrm{~S}$ & 71517.07 & 0.011112 & 0.001080 & 0.000107 & 0.003099 & 0.002713 \\
2 & $\mathrm{~S}$ & 113223.26 & 0.065395 & 0.008555 & 0.005510 & 0.017477 & 0.036065 \\
3 & $\mathrm{~S}$ & 124328.82 & 0.046330 & 0.005056 & 0.002450 & 0.013523 & 0.078704 \\
4 & $\mathrm{~A}$ & 130000.47 & 0.076123 & 0.012807 & 0.007946 & 0.024218 & 0.209863 \\
5 & $\mathrm{~S}$ & 147607.11 & 0.102151 & 0.017659 & 0.016404 & 0.028347 & 0.008013 \\
6 & $\mathrm{~S}$ & 150955.83 & 0.045439 & 0.004820 & 0.002342 & 0.013091 & 0.207442 \\
7 & $\mathrm{~S}$ & 176987.11 & 0.138696 & 0.036624 & 0.035666 & 0.038798 & 0.011464 \\
8 & $\mathrm{~S}$ & 178151.03 & 0.122330 & 0.020303 & 0.025855 & 0.033400 & 0.034825 \\
9 & $\mathrm{~S}$ & 195577.92 & 0.149584 & 0.036575 & 0.043366 & 0.042748 & 0.047200 \\
10 & $\mathrm{~A}$ & 201509.80 & 0.084117 & 0.011303 & 0.010138 & 0.025406 & 0.232569 \\
11 & $\mathrm{~S}$ & 216241.21 & 0.102550 & 0.011888 & 0.016565 & 0.029350 & 0.296416 \\
12 & $\mathrm{~S}$ & 216933.95 & 0.153221 & 0.033238 & 0.046159 & 0.046630 & 0.033856 \\
16 & $\mathrm{~A}$ & 227918.97 & 0.089985 & 0.011581 & 0.011969 & 0.026235 & 0.202592 \\
14 & $\mathrm{~S}$ & 228379.91 & 0.132431 & 0.023074 & 0.031669 & 0.038939 & 0.354035 \\
\hline
\end{tabular}

Table 16: PIN-PMN-PT disk laterally clamped. Proposed total energy error indicator TEEL versus total energy error computed by mesh halving TEE. Modes AA (S) and AA (A). 


\section{REFERENCES}

[1] F. Fahy, Sound and structural vibration. Academic Press, 1985.

[2] H.L. Schreyer, Dispersion of semidiscretized and fully discretized systems. T. Belytschko, T.J.R. Hughes eds. Computational methods for transient analysis. Elsevier Science, 1983.

[3] F.J. Brito Castro, An error indicator based on a wave dispersion analysis for the vibration modes of isotropic elastic solids discretized by energy-orthogonal finite elements. European Journal of Computational Mechanics, 28, 171-206, 2019.

[4] M. Kimura, A. Ando (revised by D. Maurya, S. Priya), Lead zirconate titanate-based piezoceramics. K. Uchino ed. Advances piezoelectric materials. Woodhead Publishing, 2017.

[5] J. Yang, Analysis of piezoelectric devices. World Scientific, 2006.

[6] L. Luo, X. Zhao, H. Luo, Single crystal PZN-PT, PMN-PT, PSN-PT, and PIN-PT-based piezoelectric materials. K. Uchino ed. Advances piezoelectric materials. Woodhead Publishing, 2017.

[7] J. Peng, H. Luo, T. He, H. Xu, D. Lin, Elastic, dielectric, and piezoelectric characterization of $0.70 \mathrm{~Pb}\left(\mathrm{Mg}_{1 / 3} \mathrm{Nb}_{2 / 3}\right) \mathrm{O}_{3}-0.30 \mathrm{PbTiO}_{3}$ single crystals. Materials Letters, 59, 640-643, 2005.

[8] http://www.trstechnologies.com/Resources.

[9] D. Stanfield, A. Elliot, Underwater electroacoustic transducers. Peninsula Publishing, 2017.

[10] H. Allik, T.J.R. Hughes, Finite element method for piezoelectric vibration. International Journal for Numerical Methods in Engineering, 2, 151-157, 1970.

[11] B.D. Zaitsev, I.E. Kuznetsova, The energy density and power flow of acoustic waves propagating in piezoelectric materials. IEEE Transactions on Ultrasonics, Ferroelectrics, and Frequency Control, 50, 1762-1765, 2003.

[12] C.A. Felippa, B. Haugen, C. Militello, From the individual element test to finite element templates: evolution of the Patch Test. International Journal for Numerical Methods in Engineering, 38, 199-229, 1995.

[13] R.H. MacNeal, Finite elements: their design and performance. Marcel Dekker, 1994.

[14] B.A. Auld, Acoustic fields and waves in solids. Volume I. Krieger Publishing Company, 1990.

[15] M. Okrouhlík, C. Höschl, A contribution to the study of dispersive properties of onedimensional lagrangian and hermitian elements. Computers and Structures, 49, 779-795, 1993.

[16] Z.-Q. Qu, Model order reduction techniques: with applications in finite element analysis. Springer, 2004.

[17] R.A. Horn, C.R. Johnson, Matrix analysis. Cambridge University Press, 1992.

[18] R.B. King, Beyond the quartic equation. Birkhäuser, 1996. 
[19] V. Laude, A. Reinhardt, A. Khelif, Equality of the energy and group velocities of bulk acoustic waves in piezoelectric media. IEEE Transactions on Ultrasonics, Ferroelectrics, and Frequency Control, 52, 1869-1871, 2005.

[20] D. Royer, E. Dieulesaint, Elastic waves in solids I. Springer, 2000.

[21] L. Brillouin, Wave propagation in periodic structures. Dover Publications, 2003.

[22] J. Barlow, More on optimal stress points-reduced integration, element distortions, and error estimation. International Journal for Numerical Methods in Engineering, 28, 14871504, 1989.

[23] M. Kaltenbacher, Numerical simulation of mechatronic sensors and actuators. Springer, 2015.

[24] J. N. Reddy, Applied functional analysis and variational methods in engineering. Krieger Publishing Company, 1991.

[25] E. Anderson, Z. Bai, C. Bischof, S. Blackford, J. Demmel, J. Dongarra, J. Du Croz, A. Greenbaum, S. Hammarling, A. McKenney, D. Sorensen, LAPACK user's guide. Society for Industrial and Applied Mathematics, 1999.

[26] B.D. Zaitsev, I.E. Kuznetsova, A.A. Teplykh, Definition of electromechanical coupling coefficient of bulk acoustic waves from energy consideration. IEEE Symposium on Ultrasonics, 1467-1470, 2003.

[27] F. Lowrie, M. Cain, M. Stewart, Finite element modelling of electroceramics. NPL Report CMMT(A)150, 1999.

[28] M.S. Vijaya, Piezoelectric materials and devices. CRC Press, 2013.

[29] A. Loussert, J-C. Debus, G. Vanderborck, Studying the behavior of piezoelectric single crystals for sonar using ATILA. K. Uchino ed. Applications of ATILA FEM software to smart materials. Woodhead Publishing, 2013.

[30] M. Petyt, Introduction to finite element vibration analysis. Cambridge University Press, 2010. 
Appendix 1. Materials constants for selected piezoelectric media.

\begin{tabular}{crrrrrr}
\hline$C_{I J}^{E}(\mathrm{GPa})$ & PZT-5H & PZT-8 & PZT-5A & PMN-30PT & PMN.31PT & PIN-PMN-PT \\
\hline 11 & 126.0 & 137.0 & 121.0 & 160.4 & 124.0 & 119.0 \\
12 & 79.1 & 69.9 & 75.9 & 149.6 & 111.0 & 105.0 \\
13 & 83.9 & 71.1 & 75.4 & 124.0 & 104.0 & 104.0 \\
22 & 126.0 & 137.0 & 121.0 & 160.4 & 124.0 & 119.0 \\
23 & 83.9 & 71.1 & 75.4 & 124.0 & 104.0 & 104.0 \\
33 & 117.0 & 123.0 & 111.0 & 120.0 & 108.0 & 114.0 \\
44 & 23.0 & 31.3 & 21.1 & 53.8 & 63.0 & 66.0 \\
55 & 23.0 & 31.3 & 21.1 & 53.8 & 63.0 & 66.0 \\
66 & 23.5 & 33.6 & 22.6 & 28.7 & 35.0 & 25.0 \\
\hline$\varepsilon_{i j}^{S} x 10^{8}(\mathrm{C} / \mathrm{Vm})$ & PZT-5H & PZT-8 & PZT-5A & PMN-30PT & PMN.31PT & PIN-PMN-PT \\
\hline 11 & 1.505 & 0.797 & 0.811 & 4.394 & 1.186 & 1.062 \\
22 & 1.505 & 0.797 & 0.811 & 4.394 & 1.186 & 1.062 \\
33 & 1.302 & 0.514 & 0.735 & 1.227 & 0.806 & 0.645 \\
\hline$e_{i J}\left(\mathrm{C} / \mathrm{m}^{2}\right)$ & PZT-5H & PZT-8 & PZT-5A & PMN-30PT & PMN.31PT & PIN-PMN-PT \\
\hline 15 & 17.00 & 10.40 & 12.30 & 31.84 & 10.30 & 6.90 \\
24 & 17.00 & 10.40 & 12.30 & 31.84 & 10.30 & 6.90 \\
31 & -6.50 & -4.00 & -5.40 & -5.22 & -3.90 & -4.80 \\
32 & -6.50 & -4.00 & -5.40 & -5.22 & -3.90 & -4.80 \\
33 & 23.30 & 13.20 & 15.80 & 30.40 & 22.30 & 18.60 \\
\hline$\rho\left(\mathrm{kg} / \mathrm{m}^{3}\right)$ & 7500 & 7600 & 7750 & 7740 & 7740 & 8000 \\
\hline $\mathrm{T}$ & & & &
\end{tabular}

Table A1.1: Material constants for PZT piezoceramics and PMN-PT $<001>$ poled single crystals. 
Appendix 2. Detailed results of the numerical dispersion analysis carried out for media and meshes selected.

\begin{tabular}{|c|c|c|c|c|c|c|c|}
\hline & & \multicolumn{3}{|c|}{ HE20 } & \multicolumn{3}{|c|}{ HE20 } \\
\hline$m_{1}^{M}$ & & PZT-5H & PZT-8 & PZT-5A & PMN-30PT & PMN-31PT & PIN-PMN-PT \\
\hline \multirow[t]{3}{*}{ M-Q1 } & SS & 0.1802 & 0.1800 & 0.1799 & 0.1796 & 0.1798 & 0.1798 \\
\hline & FS & 0.1799 & 0.1801 & 0.1802 & 0.1797 & 0.1797 & 0.1797 \\
\hline & $\mathrm{L}$ & 0.1791 & 0.1791 & 0.1791 & 0.1788 & 0.1788 & 0.1788 \\
\hline \multirow[t]{3}{*}{ M-Q2 } & SS & 0.1756 & 0.1754 & 0.1753 & 0.1749 & 0.1751 & 0.1751 \\
\hline & FS & 0.1753 & 0.1755 & 0.1756 & 0.1752 & 0.1751 & 0.1752 \\
\hline & $\mathrm{L}$ & 0.1745 & 0.1745 & 0.1745 & 0.1742 & 0.1742 & 0.1742 \\
\hline \multirow[t]{3}{*}{ M-Q3 } & $\mathrm{SS}$ & 0.1710 & 0.1709 & 0.1707 & 0.1703 & 0.1706 & 0.1705 \\
\hline & FS & 0.1711 & 0.1713 & 0.1714 & 0.1709 & 0.1709 & 0.1709 \\
\hline & $\mathrm{L}$ & 0.1706 & 0.1707 & 0.1706 & 0.1704 & 0.1704 & 0.1704 \\
\hline \multirow[t]{3}{*}{ M-Q4 } & SS & 0.1559 & 0.1557 & 0.1557 & 0.1552 & 0.1554 & 0.1554 \\
\hline & FS & 0.1556 & 0.1558 & 0.1558 & 0.1554 & 0.1554 & 0.1554 \\
\hline & $\mathrm{L}$ & 0.1548 & 0.1549 & 0.1548 & 0.1545 & 0.1545 & 0.1545 \\
\hline \multirow[t]{4}{*}{ M-Q5 } & $\mathrm{SS}$ & 0.1963 & 0.1962 & 0.1961 & 0.1956 & 0.1957 & 0.1957 \\
\hline & FS & 0.1961 & 0.1962 & 0.1963 & 0.1960 & 0.1959 & 0.1960 \\
\hline & $\mathrm{L}$ & 0.1951 & 0.1952 & 0.1952 & 0.1948 & 0.1948 & 0.1948 \\
\hline & & \multicolumn{3}{|c|}{ PE15 } & \multicolumn{3}{|c|}{ PE15 } \\
\hline$m_{1}^{M}$ & & PZT-5H & PZT-8 & PZT-5A & PMN-30PT & PMN-31PT & PIN-PMN-PT \\
\hline \multirow[t]{3}{*}{$\mathrm{M}-\mathrm{T} 1$} & SS & 0.1835 & 0.1834 & 0.1833 & 0.1826 & 0.1829 & 0.1829 \\
\hline & FS & 0.1834 & 0.1836 & 0.1837 & 0.1834 & 0.1834 & 0.1833 \\
\hline & $\mathrm{L}$ & 0.1827 & 0.1828 & 0.1828 & 0.1826 & 0.1826 & 0.1825 \\
\hline \multirow[t]{3}{*}{ M-T2 } & SS & $0 . .1804$ & 0.1804 & 0.1802 & 0.1799 & 0.1803 & 0.1801 \\
\hline & FS & 0.1806 & 0.1808 & 0.1808 & 0.1804 & 0.1805 & 0.1804 \\
\hline & $\mathrm{L}$ & 0.1799 & 0.1800 & 0.1800 & 0.1798 & 0.1798 & 0.1798 \\
\hline \multirow[t]{3}{*}{ M-T3 } & SS & 0.1779 & 0.1778 & 0.1777 & 0.1761 & 0.1766 & 0.1767 \\
\hline & FS & 0.1780 & 0.1782 & 0.1782 & 0.1779 & 0.1779 & 0.1779 \\
\hline & $\mathrm{L}$ & 0.1774 & 0.1775 & 0.1774 & 0.1772 & 0.1772 & 0.1772 \\
\hline \multirow[t]{3}{*}{ M-T4 } & $\mathrm{SS}$ & 0.1542 & 0.1541 & 0.1540 & 0.1533 & 0.1535 & 0.1536 \\
\hline & FS & 0.1540 & 0.1542 & 0.1542 & 0.1538 & 0.1539 & 0.1538 \\
\hline & $\mathrm{L}$ & 0.1534 & 0.1535 & 0.1535 & 0.1533 & 0.1533 & 0.1532 \\
\hline \multirow[t]{3}{*}{ M-T5 } & SS & 0.2062 & 0.2061 & 0.2060 & 0.2051 & 0.2053 & 0.2053 \\
\hline & FS & 0.2062 & 0.2063 & 0.2064 & 0.2061 & 0.2061 & 0.2061 \\
\hline & $\mathrm{L}$ & 0.2052 & 0.2053 & 0.2052 & 0.2050 & 0.2050 & 0.2049 \\
\hline
\end{tabular}

Table A2.1: Piezoelectric ceramics PZT and PMN-PT $<001>$ poled single crystals. Mean values of the first reference dimensionless wave number computed over the range of azimuthal and polar angles. 
Francisco J. Brito Castro

\begin{tabular}{|c|c|c|c|c|c|c|c|}
\hline & & \multicolumn{3}{|c|}{ HE20 } & \multicolumn{3}{|c|}{ HE20 } \\
\hline$m_{2}^{M}$ & & PZT-5H & PZT-8 & PZT-5A & PMN-30PT & PMN-31PT & PIN-PMN-PT \\
\hline \multirow[t]{3}{*}{ M-Q1 } & SS & 0.2642 & 0.2635 & 0.2633 & 0.2620 & 0.2624 & 0.2624 \\
\hline & FS & 0.2631 & 0.2638 & 0.2641 & 0.2627 & 0.2626 & 0.2626 \\
\hline & $\mathrm{L}$ & 0.2604 & 0.2607 & 0.2605 & 0.2594 & 0.2594 & 0.2595 \\
\hline \multirow[t]{3}{*}{ M-Q2 } & SS & 0.2574 & 0.2569 & 0.2565 & 0.2551 & 0.2555 & 0.2555 \\
\hline & FS & 0.2565 & 0.2571 & 0.2574 & 0.2561 & 0.2560 & 0.2560 \\
\hline & $\mathrm{L}$ & 0.2536 & 0.2539 & 0.2537 & 0.2523 & 0.2524 & 0.2525 \\
\hline \multirow[t]{3}{*}{ M-Q3 } & SS & 0.2499 & 0.2497 & 0.2490 & 0.2478 & 0.2485 & 0.2484 \\
\hline & FS & 0.2502 & 0.2507 & 0.2511 & 0.2495 & 0.2495 & 0.2495 \\
\hline & L & 0.2484 & 0.2486 & 0.2485 & 0.2477 & 0.2478 & 0.2478 \\
\hline \multirow[t]{3}{*}{ M-Q4 } & SS & 0.2287 & 0.2281 & 0.2279 & 0.2265 & 0.2269 & 0.2268 \\
\hline & FS & 0.2274 & 0.2282 & 0.2283 & 0.2268 & 0.2269 & 0.2268 \\
\hline & $\mathrm{L}$ & 0.2247 & 0.2252 & 0.2249 & 0.2234 & 0.2237 & 0.2238 \\
\hline \multirow[t]{4}{*}{ M-Q5 } & SS & 0.2877 & 0.2873 & 0.2870 & 0.2853 & 0.2856 & 0.2855 \\
\hline & FS & 0.2870 & 0.2873 & 0.2878 & 0.2867 & 0.2866 & 0.2867 \\
\hline & $\mathrm{L}$ & 0.2838 & 0.2841 & 0.2839 & 0.2822 & 0.2823 & 0.2823 \\
\hline & & \multicolumn{3}{|c|}{ PE15 } & \multicolumn{3}{|c|}{ PE15 } \\
\hline$m_{2}^{M}$ & & PZT-5H & PZT-8 & PZT-5A & PMN-30PT & PMN-31PT & PIN-PMN-PT \\
\hline \multirow[t]{3}{*}{ M-T1 } & SS & 0.2675 & 0.2672 & 0.2669 & 0.2647 & 0.2655 & 0.2655 \\
\hline & FS & 0.2671 & 0.2676 & 0.2678 & 0.2668 & 0.2669 & 0.2668 \\
\hline & $\mathrm{L}$ & 0.2650 & 0.2652 & 0.2651 & 0.2645 & 0.2644 & 0.2643 \\
\hline \multirow[t]{3}{*}{ M-T2 } & SS & 0.2625 & 0.2624 & 0.2619 & 0.2608 & 0.2619 & 0.2614 \\
\hline & FS & 0.2629 & 0.2634 & 0.2636 & 0.2624 & 0.2625 & 0.2624 \\
\hline & $\mathrm{L}$ & 0.2610 & 0.2612 & 0.2611 & 0.2603 & 0.2603 & 0.2603 \\
\hline \multirow[t]{3}{*}{ M-T3 } & SS & 0.2589 & 0.2588 & 0.2583 & 0.2544 & 0.2554 & 0.2557 \\
\hline & FS & 0.2591 & 0.2597 & 0.2598 & 0.2589 & 0.2589 & 0.2588 \\
\hline & $\mathrm{L}$ & 0.2572 & 0.2575 & 0.2573 & 0.2569 & 0.2569 & 0.2568 \\
\hline \multirow[t]{3}{*}{ M-T4 } & SS & 0.2246 & 0.2244 & 0.2242 & 0.2222 & 0.2228 & 0.2228 \\
\hline & FS & 0.2240 & 0.2245 & 0.2246 & 0.2234 & 0.2236 & 0.2234 \\
\hline & $\mathrm{L}$ & 0.2222 & 0.2225 & 0.2223 & 0.2214 & 0.2216 & 0.2215 \\
\hline \multirow[t]{3}{*}{ M-T5 } & SS & 0.3007 & 0.3005 & 0.3001 & 0.2972 & 0.2980 & 0.2978 \\
\hline & FS & 0.3005 & 0.3009 & 0.3012 & 0.3004 & 0.3004 & 0.3004 \\
\hline & $\mathrm{L}$ & 0.2974 & 0.2978 & 0.2976 & 0.2965 & 0.2964 & 0.2961 \\
\hline
\end{tabular}

Table A2.2: Piezoelectric ceramics PZT and PMN-PT $<001>$ poled single crystals. Mean values of the second reference dimensionless wave number computed over the range of azimuthal and polar angles. 
Francisco J. Brito Castro

\begin{tabular}{|c|c|c|c|c|c|c|c|}
\hline & & \multicolumn{3}{|c|}{ HE20 } & \multicolumn{3}{|c|}{ HE20 } \\
\hline$e_{T 1}^{R M S}$ & & PZT-5H & PZT-8 & PZT-5A & PMN-30PT & PMN-31PT & PIN-PMN-PT \\
\hline \multirow[t]{3}{*}{ M-Q1 } & SS & 0.003111 & 0.002951 & 0.002747 & 0.002781 & 0.002615 & 0.002589 \\
\hline & FS & 0.002440 & 0.002590 & 0.002839 & 0.002930 & 0.002996 & 0.002967 \\
\hline & $\mathrm{L}$ & 0.005436 & 0.005160 & 0.005353 & 0.006342 & 0.006091 & 0.006031 \\
\hline \multirow[t]{3}{*}{$\mathrm{M}-\mathrm{Q} 2$} & SS & 0.003213 & 0.003019 & 0.002903 & 0.003171 & 0.003015 & 0.003008 \\
\hline & FS & 0.002519 & 0.002608 & 0.002856 & 0.002773 & 0.002799 & 0.002787 \\
\hline & $\mathrm{L}$ & 0.005893 & 0.005474 & 0.005792 & 0.007055 & 0.006668 & 0.006613 \\
\hline \multirow[t]{3}{*}{ M-Q3 } & SS & 0.003109 & 0.002987 & 0.002823 & 0.002940 & 0.002816 & 0.002774 \\
\hline & FS & 0.002493 & 0.002586 & 0.002803 & 0.002673 & 0.002728 & 0.002738 \\
\hline & $\mathrm{L}$ & 0.004525 & 0.004330 & 0.004461 & 0.005224 & 0.004918 & 0.004890 \\
\hline \multirow[t]{3}{*}{ M-Q4 } & SS & 0.003788 & 0.002963 & 0.002673 & 0.004246 & 0.003890 & 0.003829 \\
\hline & FS & 0.003075 & 0.003603 & 0.004038 & 0.002462 & 0.002583 & 0.002598 \\
\hline & $\mathrm{L}$ & 0.006038 & 0.005626 & 0.005959 & 0.007375 & 0.006487 & 0.006303 \\
\hline \multirow[t]{4}{*}{ M-Q5 } & SS & 0.002391 & 0.002670 & 0.002647 & 0.002729 & 0.002731 & 0.002728 \\
\hline & FS & 0.002962 & 0.002702 & 0.002738 & 0.002819 & 0.002793 & 0.002717 \\
\hline & $\mathrm{L}$ & 0.006349 & 0.005853 & 0.006163 & 0.007449 & 0.007278 & 0.007277 \\
\hline & & \multicolumn{3}{|c|}{ PE15 } & \multicolumn{3}{|c|}{ PE15 } \\
\hline$e_{T 1}^{R M S}$ & & PZT-5H & PZT-8 & PZT-5A & PMN-30PT & PMN-31PT & PIN-PMN-PT \\
\hline \multirow[t]{3}{*}{ M-T1 } & SS & 0.005204 & 0.004864 & 0.005081 & 0.005872 & 0.005807 & 0.005738 \\
\hline & FS & 0.004828 & 0.004707 & 0.004887 & 0.004947 & 0.004918 & 0.005011 \\
\hline & $\mathrm{L}$ & 0.006325 & 0.005857 & 0.006344 & 0.007948 & 0.007223 & 0.006921 \\
\hline \multirow[t]{3}{*}{ M-T2 } & SS & 0.005582 & 0.005380 & 0.005476 & 0.006839 & 0.006869 & 0.006442 \\
\hline & FS & 0.005509 & 0.005401 & 0.005555 & 0.005756 & 0.005776 & 0.005813 \\
\hline & $\mathrm{L}$ & 0.006614 & 0.006188 & 0.006638 & 0.010839 & 0.009606 & 0.008615 \\
\hline \multirow[t]{3}{*}{ M-T3 } & SS & 0.005859 & 0.005642 & 0.005765 & 0.005729 & 0.005891 & 0.005936 \\
\hline & FS & 0.006131 & 0.005981 & 0.006151 & 0.006018 & 0.005922 & 0.006063 \\
\hline & $\mathrm{L}$ & 0.009322 & 0.008403 & 0.009355 & 0.009636 & 0.008012 & 0.008553 \\
\hline \multirow[t]{3}{*}{ M-T4 } & SS & 0.004190 & 0.003745 & 0.003880 & 0.004819 & 0.004663 & 0.004619 \\
\hline & FS & 0.003617 & 0.003699 & 0.003907 & 0.003422 & 0.003417 & 0.003452 \\
\hline & $\mathrm{L}$ & 0.005639 & 0.005138 & 0.005625 & 0.007236 & 0.006429 & 0.006180 \\
\hline \multirow[t]{3}{*}{ M-T5 } & SS & 0.006740 & 0.006416 & 0.006742 & 0.008203 & 0.008201 & 0.008165 \\
\hline & FS & 0.007562 & 0.007175 & 0.007438 & 0.007272 & 0.007028 & 0.007186 \\
\hline & $\mathrm{L}$ & 0.007694 & 0.007187 & 0.007731 & 0.009288 & 0.008404 & 0.008053 \\
\hline
\end{tabular}

Table A2.3: Piezoelectric ceramics PZT and PMN-PT $<001>$ poled single crystals. Root-mean-square values of the first reference percentage of total energy error computed over the range of azimuthal and polar angles. 
Francisco J. Brito Castro

\begin{tabular}{|c|c|c|c|c|c|c|c|}
\hline & & \multicolumn{3}{|c|}{ HE20 } & \multicolumn{3}{|c|}{ HE20 } \\
\hline$e_{T 2}^{R M S}$ & & PZT-5H & PZT-8 & PZT-5A & PMN-30PT & PMN-31PT & PIN-PMN-PT \\
\hline \multirow[t]{3}{*}{ M-Q1 } & SS & 0.013381 & 0.012952 & 0.011943 & 0.011387 & 0.010701 & 0.010581 \\
\hline & FS & 0.010521 & 0.011113 & 0.012135 & 0.012881 & 0.013263 & 0.013028 \\
\hline & $\mathrm{L}$ & 0.026983 & 0.025343 & 0.026457 & 0.032994 & 0.031433 & 0.031055 \\
\hline \multirow[t]{3}{*}{ M-Q2 } & $\mathrm{SS}$ & 0.013659 & 0.013025 & 0.012411 & 0.012765 & 0.012112 & 0.012084 \\
\hline & FS & 0.010365 & 0.010741 & 0.011795 & 0.011805 & 0.012004 & 0.011841 \\
\hline & $\mathrm{L}$ & 0.031094 & 0.027931 & 0.030371 & 0.040943 & 0.037724 & 0.037389 \\
\hline \multirow[t]{3}{*}{ M-Q3 } & $\mathrm{SS}$ & 0.013164 & 0.012886 & 0.011972 & 0.011998 & 0.011539 & 0.011337 \\
\hline & FS & 0.010731 & 0.011173 & 0.012022 & 0.011668 & 0.012057 & 0.012025 \\
\hline & $\mathrm{L}$ & 0.022453 & 0.021275 & 0.022036 & 0.027481 & 0.025402 & 0.025162 \\
\hline \multirow[t]{3}{*}{ M-Q4 } & $\mathrm{SS}$ & 0.015347 & 0.011635 & 0.009988 & 0.017049 & 0.015701 & 0.015482 \\
\hline & FS & 0.012378 & 0.014993 & 0.016828 & 0.009974 & 0.010623 & 0.010723 \\
\hline & $\mathrm{L}$ & 0.033683 & 0.030260 & 0.032918 & 0.047561 & 0.037925 & 0.036204 \\
\hline \multirow[t]{4}{*}{ M-Q5 } & $\mathrm{SS}$ & 0.010234 & 0.011658 & 0.011535 & 0.010967 & 0.010975 & 0.010923 \\
\hline & FS & 0.012743 & 0.011603 & 0.011675 & 0.011987 & 0.011861 & 0.011295 \\
\hline & $\mathrm{L}$ & 0.033641 & 0.029885 & 0.032162 & 0.042194 & 0.041363 & 0.041531 \\
\hline & & \multicolumn{3}{|c|}{ PE15 } & \multicolumn{3}{|c|}{ PE15 } \\
\hline$e_{T 2}^{R M S}$ & & PZT-5H & PZT-8 & PZT-5A & PMN-30PT & PMN-31PT & PIN-PMN-PT \\
\hline \multirow[t]{3}{*}{ M-T1 } & SS & 0.021343 & 0.020127 & 0.020836 & 0.022352 & 0.022299 & 0.022229 \\
\hline & FS & 0.019872 & 0.019338 & 0.020127 & 0.020431 & 0.020349 & 0.020722 \\
\hline & $\mathrm{L}$ & 0.028526 & 0.025765 & 0.028627 & 0.039510 & 0.034663 & 0.032725 \\
\hline \multirow[t]{3}{*}{ M-T2 } & $\mathrm{SS}$ & 0.021951 & 0.021364 & 0.021493 & 0.025771 & 0.026720 & 0.024810 \\
\hline & FS & 0.022818 & 0.022377 & 0.023026 & 0.023755 & 0.023791 & 0.023963 \\
\hline & $\mathrm{L}$ & 0.030466 & 0.028074 & 0.030559 & 0.043109 & 0.037112 & 0.035260 \\
\hline \multirow[t]{3}{*}{ M-T3 } & $\mathrm{SS}$ & 0.023094 & 0.022519 & 0.022695 & 0.020830 & 0.021604 & 0.021877 \\
\hline & FS & 0.025246 & 0.024654 & 0.025354 & 0.024875 & 0.024519 & 0.025062 \\
\hline & $\mathrm{L}$ & 0.044886 & 0.038575 & 0.045003 & 0.094928 & 0.073876 & 0.079793 \\
\hline \multirow[t]{3}{*}{ M-T4 } & $\mathrm{SS}$ & 0.017826 & 0.015975 & 0.016563 & 0.018830 & 0.018301 & 0.018200 \\
\hline & FS & 0.014637 & 0.015075 & 0.015944 & 0.013936 & 0.013951 & 0.014109 \\
\hline & $\mathrm{L}$ & 0.028755 & 0.025083 & 0.028547 & 0.040516 & 0.033460 & 0.031638 \\
\hline \multirow[t]{3}{*}{ M-T5 } & $\mathrm{SS}$ & 0.027293 & 0.026275 & 0.027327 & 0.031205 & 0.031590 & 0.031633 \\
\hline & FS & 0.031278 & 0.029741 & 0.030795 & 0.030180 & 0.029226 & 0.029860 \\
\hline & $\mathrm{L}$ & 0.034614 & 0.031055 & 0.034500 & 0.046293 & 0.040770 & 0.038983 \\
\hline
\end{tabular}

Table A2.4: Piezoelectric ceramics PZT and PMN-PT $<001>$ poled single crystals. Root-mean-square values of the second reference percentage of total energy error computed over the range of azimuthal and polar angles. 
Francisco J. Brito Castro

\begin{tabular}{|c|c|c|c|c|c|c|c|}
\hline & & \multicolumn{3}{|c|}{ HE20 } & \multicolumn{3}{|c|}{ HE20 } \\
\hline$e_{T 1}^{S U P}$ & & PZT-5H & PZT-8 & PZT-5A & PMN-30PT & PMN-31PT & PIN-PMN-PT \\
\hline \multirow[t]{3}{*}{ M-Q1 } & SS & 0.004946 & 0.005104 & 0.004941 & 0.004871 & 0.004458 & 0.004589 \\
\hline & FS & 0.004842 & 0.005008 & 0.004932 & 0.004646 & 0.004730 & 0.004652 \\
\hline & $\mathrm{L}$ & 0.010287 & 0.009793 & 0.010192 & 0.011434 & 0.010889 & 0.010801 \\
\hline \multirow[t]{3}{*}{ M-Q2 } & SS & 0.006064 & 0.005429 & 0.006061 & 0.007097 & 0.006597 & 0.006802 \\
\hline & FS & 0.004773 & 0.004942 & 0.005058 & 0.004894 & 0.005017 & 0.005071 \\
\hline & $\mathrm{L}$ & 0.011419 & 0.010569 & 0.011289 & 0.012986 & 0.012092 & 0.011983 \\
\hline \multirow[t]{3}{*}{ M-Q3 } & SS & 0.006280 & 0.006588 & 0.006278 & 0.006705 & 0.006410 & 0.006537 \\
\hline & FS & 0.007473 & 0.007473 & 0.007473 & 0.007473 & 0.007473 & 0.007473 \\
\hline & $\mathrm{L}$ & 0.012429 & 0.011731 & 0.012293 & 0.013516 & 0.012878 & 0.012787 \\
\hline \multirow[t]{3}{*}{ M-Q4 } & SS & 0.006267 & 0.005117 & 0.004463 & 0.006485 & 0.005851 & 0.005693 \\
\hline & FS & 0.005446 & 0.005645 & 0.006180 & 0.004974 & 0.004977 & 0.004905 \\
\hline & $\mathrm{L}$ & 0.012539 & 0.011542 & 0.012451 & 0.014192 & 0.012454 & 0.012105 \\
\hline \multirow[t]{4}{*}{ M-Q5 } & SS & 0.004866 & 0.005098 & 0.004990 & 0.005691 & 0.005962 & 0.006050 \\
\hline & FS & 0.005615 & 0.005225 & 0.005453 & 0.005857 & 0.005873 & 0.005952 \\
\hline & $\mathrm{L}$ & 0.011288 & 0.010573 & 0.011035 & 0.012766 & 0.012186 & 0.012215 \\
\hline & & \multicolumn{3}{|c|}{ PE15 } & \multicolumn{3}{|c|}{ PE15 } \\
\hline$e_{T 1}^{S U P}$ & & PZT-5H & PZT-8 & PZT-5A & PMN-30PT & PMN-31PT & PIN-PMN-PT \\
\hline \multirow[t]{3}{*}{ M-T1 } & SS & 0.017162 & 0.016391 & 0.017158 & 0.017801 & 0.018963 & 0.018396 \\
\hline & FS & 0.010875 & 0.010875 & 0.010875 & 0.010875 & 0.010960 & 0.011192 \\
\hline & $\mathrm{L}$ & 0.022223 & 0.019957 & 0.022213 & 0.033930 & 0.033048 & 0.030928 \\
\hline \multirow[t]{3}{*}{ M-T2 } & SS & 0.017410 & 0.017854 & 0.017448 & 0.020036 & 0.021348 & 0.019359 \\
\hline & FS & 0.017457 & 0.017457 & 0.017457 & 0.017457 & 0.017457 & 0.017468 \\
\hline & $\mathrm{L}$ & 0.030500 & 0.028698 & 0.030492 & 0.060299 & 0.050362 & 0.046393 \\
\hline \multirow[t]{3}{*}{ M-T3 } & SS & 0.018419 & 0.018323 & 0.018417 & 0.016684 & 0.017354 & 0.017172 \\
\hline & FS & 0.020527 & 0.020527 & 0.020527 & 0.020527 & 0.020527 & 0.020527 \\
\hline & $\mathrm{L}$ & 0.051384 & 0.044223 & 0.051347 & 0.058955 & 0.047983 & 0.053773 \\
\hline \multirow[t]{3}{*}{ M-T4 } & SS & 0.017173 & 0.016383 & 0.017168 & 0.017784 & 0.018966 & 0.018397 \\
\hline & FS & 0.010875 & 0.010875 & 0.010875 & 0.010875 & 0.010875 & 0.010875 \\
\hline & $\mathrm{L}$ & 0.022248 & 0.019952 & 0.022238 & 0.033900 & 0.033078 & 0.030923 \\
\hline \multirow[t]{3}{*}{ M-T5 } & SS & 0.017177 & 0.016386 & 0.017172 & 0.017794 & 0.018978 & 0.018397 \\
\hline & FS & 0.013150 & 0.012228 & 0.012845 & 0.014029 & 0.014507 & 0.014523 \\
\hline & $\mathrm{L}$ & 0.022229 & 0.019925 & 0.022219 & 0.033913 & 0.033080 & 0.030943 \\
\hline
\end{tabular}

Table A2.5: Piezoelectric ceramics PZT and PMN-PT $<001>$ poled single crystals. Sup-norm of the first reference percentage of total energy error computed over the range of azimuthal and polar angles. 
Francisco J. Brito Castro

\begin{tabular}{|c|c|c|c|c|c|c|c|}
\hline & & \multicolumn{3}{|c|}{ HE20 } & \multicolumn{3}{|c|}{ HE20 } \\
\hline$e_{T 2}^{S U P}$ & & PZT-5H & PZT-8 & PZT-5A & PMN-30PT & PMN-31PT & PIN-PMN-PT \\
\hline \multirow[t]{3}{*}{ M-Q1 } & SS & 0.023199 & 0.024125 & 0.023117 & 0.020298 & 0.020892 & 0.020491 \\
\hline & FS & 0.023037 & 0.023882 & 0.023467 & 0.022050 & 0.022476 & 0.022309 \\
\hline & $\mathrm{L}$ & 0.053215 & 0.050128 & 0.052505 & 0.060786 & 0.057137 & 0.056547 \\
\hline \multirow[t]{3}{*}{$\mathrm{M}-\mathrm{Q} 2$} & SS & 0.025362 & 0.024742 & 0.025350 & 0.029209 & 0.027160 & 0.027970 \\
\hline & FS & 0.021637 & 0.022538 & 0.022121 & 0.022383 & 0.023682 & 0.024075 \\
\hline & $\mathrm{L}$ & 0.066331 & 0.057934 & 0.065265 & 0.095463 & 0.078166 & 0.083769 \\
\hline \multirow[t]{3}{*}{ M-Q3 } & SS & 0.030076 & 0.031723 & 0.030035 & 0.027694 & 0.028404 & 0.028348 \\
\hline & FS & 0.038762 & 0.038762 & 0.038762 & 0.038762 & 0.038762 & 0.038762 \\
\hline & $\mathrm{L}$ & 0.068921 & 0.063519 & 0.067837 & 0.079279 & 0.072882 & 0.072098 \\
\hline \multirow[t]{3}{*}{ M-Q4 } & SS & 0.026212 & 0.020792 & 0.016989 & 0.026779 & 0.024285 & 0.023593 \\
\hline & FS & 0.025050 & 0.025642 & 0.025882 & 0.023777 & 0.023850 & 0.023456 \\
\hline & $\mathrm{L}$ & 0.077414 & 0.067116 & 0.076350 & 0.100073 & 0.075566 & 0.071586 \\
\hline \multirow[t]{4}{*}{ M-Q5 } & SS & 0.023427 & 0.024327 & 0.023882 & 0.023406 & 0.024710 & 0.024995 \\
\hline & FS & 0.023853 & 0.021739 & 0.022733 & 0.024290 & 0.024316 & 0.024581 \\
\hline & $\mathrm{L}$ & 0.062078 & 0.056119 & 0.060119 & 0.073130 & 0.068866 & 0.069332 \\
\hline & & \multicolumn{3}{|c|}{ PE15 } & \multicolumn{3}{|c|}{ PE15 } \\
\hline$e_{T 2}^{S U P}$ & & $\mathrm{PZT}-5 \mathrm{H}$ & PZT-8 & PZT-5A & PMN-30PT & PMN-31PT & PIN-PMN-PT \\
\hline \multirow[t]{3}{*}{ M-T1 } & SS & 0.068776 & 0.067332 & 0.068762 & 0.068879 & 0.068204 & 0.068301 \\
\hline & FS & 0.045641 & 0.045641 & 0.045641 & 0.045641 & 0.046194 & 0.046726 \\
\hline & $\mathrm{L}$ & 0.109239 & 0.092908 & 0.109166 & 0.217268 & 0.193448 & 0.182894 \\
\hline \multirow[t]{3}{*}{ M-T2 } & SS & 0.063741 & 0.064894 & 0.063723 & 0.076098 & 0.082671 & 0.075965 \\
\hline & FS & 0.076963 & 0.076963 & 0.076963 & 0.076963 & 0.078231 & 0.079146 \\
\hline & $\mathrm{L}$ & 0.193846 & 0.170351 & 0.193762 & 0.211705 & 0.171666 & 0.183435 \\
\hline \multirow[t]{3}{*}{ M-T3 } & SS & 0.069605 & 0.071006 & 0.069599 & 0.058999 & 0.062114 & 0.062565 \\
\hline & FS & 0.084933 & 0.084933 & 0.084933 & 0.084933 & 0.084933 & 0.084965 \\
\hline & $\mathrm{L}$ & 0.249039 & 0.202924 & 0.248812 & 0.735536 & 0.732378 & 0.723363 \\
\hline \multirow[t]{3}{*}{ M-T4 } & SS & 0.068788 & 0.067344 & 0.068775 & 0.068894 & 0.068208 & 0.068322 \\
\hline & FS & 0.045638 & 0.045638 & 0.045638 & 0.045638 & 0.045638 & 0.045638 \\
\hline & $\mathrm{L}$ & 0.109306 & 0.092869 & 0.109233 & 0.217447 & 0.193475 & 0.182868 \\
\hline \multirow[t]{3}{*}{ M-T5 } & SS & 0.068803 & 0.067317 & 0.068790 & 0.068830 & 0.068148 & 0.068261 \\
\hline & FS & 0.055850 & 0.051635 & 0.054473 & 0.060503 & 0.063464 & 0.062776 \\
\hline & $\mathrm{L}$ & 0.109354 & 0.092874 & 0.109111 & 0.217424 & 0.193497 & 0.183005 \\
\hline
\end{tabular}

Table A2.6: Piezoelectric ceramics PZT and PMN-PT $<001>$ poled single crystals. Sup-norm of the second reference percentage of total energy error computed over the range of azimuthal and polar angles. 
Francisco J. Brito Castro

\begin{tabular}{|c|c|c|c|c|c|c|c|}
\hline & & \multicolumn{3}{|c|}{ HE20 } & \multicolumn{3}{|c|}{ HE20 } \\
\hline$e_{p 2}^{M}$ & & PZT-5H & PZT-8 & PZT-5A & PMN-30PT & PMN-31PT & PIN-PMN-PT \\
\hline \multirow[t]{3}{*}{ M-Q1 } & SS & 1.00355 & 1.00294 & 1.00301 & 1.00716 & 1.00639 & 1.00624 \\
\hline & FS & 1.00337 & 1.00338 & 1.00370 & 1.00299 & 1.00284 & 1.00291 \\
\hline & $\mathrm{L}$ & 1.00195 & 1.00200 & 1.00197 & 1.00169 & 1.00170 & 1.00171 \\
\hline \multirow[t]{3}{*}{ M-Q2 } & SS & 1.00359 & 1.00297 & 1.00307 & 1.00724 & 1.00642 & 1.00626 \\
\hline & FS & 1.00336 & 1.00335 & 1.00367 & 1.00297 & 1.00282 & 1.00288 \\
\hline & $\mathrm{L}$ & 1.00189 & 1.00194 & 1.00191 & 1.00163 & 1.00164 & 1.00165 \\
\hline \multirow[t]{3}{*}{ M-Q3 } & SS & 1.00555 & 1.00464 & 1.00510 & 1.00876 & 1.00757 & 1.00759 \\
\hline & FS & 1.00415 & 1.00405 & 1.00438 & 1.00419 & 1.00392 & 1.00398 \\
\hline & $\mathrm{L}$ & 1.00216 & 1.00223 & 1.00218 & 1.00191 & 1.00196 & 1.00195 \\
\hline \multirow[t]{3}{*}{ M-Q4 } & SS & 1.00361 & 1.00291 & 1.00292 & 1.00768 & 1.00692 & 1.00690 \\
\hline & FS & 1.00360 & 1.00369 & 1.00402 & 1.00310 & 1.00302 & 1.00309 \\
\hline & $\mathrm{L}$ & 1.00202 & 1.00205 & 1.00204 & 1.00170 & 1.00175 & 1.00177 \\
\hline \multirow[t]{4}{*}{ M-Q5 } & SS & 1.00332 & 1.00285 & 1.00298 & 1.00674 & 1.00595 & 1.00573 \\
\hline & FS & 1.00331 & 1.00315 & 1.00348 & 1.00278 & 1.00258 & 1.00262 \\
\hline & $\mathrm{L}$ & 1.00168 & 1.00175 & 1.00171 & 1.00147 & 1.00146 & 1.00147 \\
\hline & & \multicolumn{3}{|c|}{ PE15 } & \multicolumn{3}{|c|}{ PE15 } \\
\hline$e_{p 2}^{M}$ & & PZT-5H & PZT-8 & PZT-5A & PMN-30PT & PMN-31PT & PIN-PMN-PT \\
\hline \multirow[t]{3}{*}{ M-T1 } & SS & 1.00360 & 1.00308 & 1.00327 & 1.00599 & 1.00534 & 1.00540 \\
\hline & FS & 1.00335 & 1.00325 & 1.00350 & 1.00326 & 1.00310 & 1.00317 \\
\hline & $\mathrm{L}$ & 1.00185 & 1.00191 & 1.00187 & 1.00167 & 1.00168 & 1.00166 \\
\hline \multirow[t]{3}{*}{ M-T2 } & SS & 1.00436 & 1.00373 & 1.00405 & 1.00605 & 1.00530 & 1.00557 \\
\hline & FS & 1.00367 & 1.00354 & 1.00380 & 1.00377 & 1.00358 & 1.00363 \\
\hline & $\mathrm{L}$ & 1.00194 & 1.00201 & 1.00197 & 1.00176 & 1.00180 & 1.00178 \\
\hline \multirow[t]{3}{*}{ M-T3 } & SS & 1.00418 & 1.00357 & 1.00387 & 1.00766 & 1.00680 & 1.00667 \\
\hline & FS & 1.00361 & 1.00348 & 1.00373 & 1.00346 & 1.00327 & 1.00335 \\
\hline & $\mathrm{L}$ & 1.00189 & 1.00197 & 1.00192 & 1.00159 & 1.00164 & 1.00163 \\
\hline \multirow[t]{3}{*}{ M-T4 } & SS & 1.00292 & 1.00245 & 1.00255 & 1.00539 & 1.00480 & 1.00490 \\
\hline & FS & 1.00278 & 1.00278 & 1.00298 & 1.00253 & 1.00248 & 1.00254 \\
\hline & $\mathrm{L}$ & 1.00160 & 1.00163 & 1.00161 & 1.00141 & 1.00145 & 1.00144 \\
\hline \multirow[t]{3}{*}{ M-T5 } & SS & 1.00423 & 1.00368 & 1.00397 & 1.00690 & 1.00614 & 1.00620 \\
\hline & FS & 1.00421 & 1.00390 & 1.00425 & 1.00400 & 1.00367 & 1.00370 \\
\hline & $\mathrm{L}$ & 1.00195 & 1.00205 & 1.00199 & 1.00177 & 1.00177 & 1.00174 \\
\hline
\end{tabular}

Table A2.7: Piezoelectric ceramics PZT and PMN-PT $<001>$ poled single crystals. Mean values of the second reference value for the indicator of dispersion associated with the phase velocity. 
Francisco J. Brito Castro

\begin{tabular}{|c|c|c|c|c|c|c|c|}
\hline & & \multicolumn{3}{|c|}{ HE20 } & \multicolumn{3}{|c|}{ HE20 } \\
\hline$e_{g n 2}^{M}$ & & PZT-5H & PZT-8 & PZT-5A & PMN-30PT & PMN-31PT & PIN-PMN-PT \\
\hline \multirow[t]{3}{*}{ M-Q1 } & SS & 1.01756 & 1.01453 & 1.01490 & 1.03521 & 1.03136 & 1.03066 \\
\hline & FS & 1.01666 & 1.01668 & 1.01828 & 1.01476 & 1.01402 & 1.01433 \\
\hline & $\mathrm{L}$ & 1.00943 & 1.00966 & 1.00953 & 1.00808 & 1.00813 & 1.00821 \\
\hline \multirow[t]{3}{*}{ M-Q2 } & SS & 1.01770 & 1.01467 & 1.01515 & 1.03557 & 1.03153 & 1.03078 \\
\hline & FS & 1.01661 & 1.01654 & 1.01814 & 1.01465 & 1.01389 & 1.01419 \\
\hline & $\mathrm{L}$ & 1.00909 & 1.00936 & 1.00920 & 1.00768 & 1.00776 & 1.00784 \\
\hline \multirow[t]{3}{*}{ M-Q3 } & SS & 1.02754 & 1.02304 & 1.02534 & 1.04321 & 1.03735 & 1.03747 \\
\hline & FS & 1.02062 & 1.02006 & 1.02174 & 1.02075 & 1.01938 & 1.01965 \\
\hline & $\mathrm{L}$ & 1.01052 & 1.01090 & 1.01064 & 1.00924 & 1.00950 & 1.00946 \\
\hline \multirow[t]{3}{*}{ M-Q4 } & SS & 1.01778 & 1.01434 & 1.01444 & 1.03767 & 1.03389 & 1.03378 \\
\hline & FS & 1.01765 & 1.01810 & 1.01971 & 1.01519 & 1.01477 & 1.01510 \\
\hline & $\mathrm{L}$ & 1.00965 & 1.00983 & 1.00973 & 1.00782 & 1.00818 & 1.00831 \\
\hline \multirow[t]{4}{*}{ M-Q5 } & SS & 1.01647 & 1.01410 & 1.01479 & 1.03322 & 1.02933 & 1.02828 \\
\hline & FS & 1.01642 & 1.01562 & 1.01724 & 1.01381 & 1.01281 & 1.01299 \\
\hline & $\mathrm{L}$ & 1.00812 & 1.00847 & 1.00827 & 1.00695 & 1.00690 & 1.00695 \\
\hline & & \multicolumn{3}{|c|}{ PE15 } & \multicolumn{3}{|c|}{ PE15 } \\
\hline$e_{g n 2}^{M}$ & & PZT-5H & PZT-8 & PZT-5A & PMN-30PT & PMN-31PT & PIN-PMN-PT \\
\hline \multirow[t]{3}{*}{ M-T1 } & SS & 1.01745 & 1.01492 & 1.01584 & 1.02901 & 1.02590 & 1.02623 \\
\hline & FS & 1.01639 & 1.01588 & 1.01710 & 1.01585 & 1.01507 & 1.01539 \\
\hline & $\mathrm{L}$ & 1.00885 & 1.00915 & 1.00896 & 1.00785 & 1.00796 & 1.00790 \\
\hline \multirow[t]{3}{*}{ M-T2 } & SS & 1.02099 & 1.01798 & 1.01945 & 1.02925 & 1.02574 & 1.02701 \\
\hline & FS & 1.01789 & 1.01725 & 1.01850 & 1.01825 & 1.01727 & 1.01754 \\
\hline & $\mathrm{L}$ & 1.00928 & 1.00965 & 1.00940 & 1.00832 & 1.00859 & 1.00845 \\
\hline \multirow[t]{3}{*}{ M-T3 } & SS & 1.02015 & 1.01723 & 1.01862 & 1.03682 & 1.03275 & 1.03211 \\
\hline & FS & 1.01760 & 1.01695 & 1.01821 & 1.01680 & 1.01588 & 1.01625 \\
\hline & $\mathrm{L}$ & 1.00894 & 1.00936 & 1.00906 & 1.00271 & 1.00534 & 1.00456 \\
\hline \multirow[t]{3}{*}{ M-T4 } & SS & 1.01426 & 1.01194 & 1.01243 & 1.02615 & 1.02334 & 1.02384 \\
\hline & FS & 1.01358 & 1.01359 & 1.01457 & 1.01237 & 1.01210 & 1.01236 \\
\hline & $\mathrm{L}$ & 1.00764 & 1.00782 & 1.00770 & 1.00658 & 1.00683 & 1.00683 \\
\hline \multirow[t]{3}{*}{ M-T5 } & SS & 1.02035 & 1.01772 & 1.01908 & 1.03325 & 1.02968 & 1.02997 \\
\hline & FS & 1.02045 & 1.01899 & 1.02066 & 1.01931 & 1.01772 & 1.01786 \\
\hline & $\mathrm{L}$ & 1.00922 & 1.00974 & 1.00942 & 1.00818 & 1.00824 & 1.00811 \\
\hline
\end{tabular}

Table A2.8: Piezoelectric ceramics PZT and PMN-PT $<001>$ poled single crystals. Mean values of the second reference value for the indicator of dispersion associated with the normal component of the group velocity. 